\title{
OmniFiber: Integrated Fluidic Fiber Actuators for Weaving Movement based Interactions into the 'Fabric of Everyday Life'
}

\author{
Ozgun Kilic Afsar ${ }^{1,2}$ \\ Ali Shtarbanov ${ }^{2}$ \\ Hila Mor ${ }^{2}$ \\ Karen Modrei \\ Seung Hee Jeong ${ }^{3}$ \\ Klas Hjort ${ }^{3}$ \\ Ken Nakagaki ${ }^{2}$ \\ Jack Forman $^{2}$ \\ ${ }^{1}$ KTH Royal Institute of Technology, Sweden \\ ${ }^{2}$ MIT Media Lab, USA \\ Hiroshi Ishii ${ }^{2}$
}

\{ozgunk,khook\}@kth.se

\{ozgun,alims,hilamor,ken_n,jackform,ishii\}@media.mit.edu

\{seunghee.jeong,klas.hjort\}@angstrom.uu.se
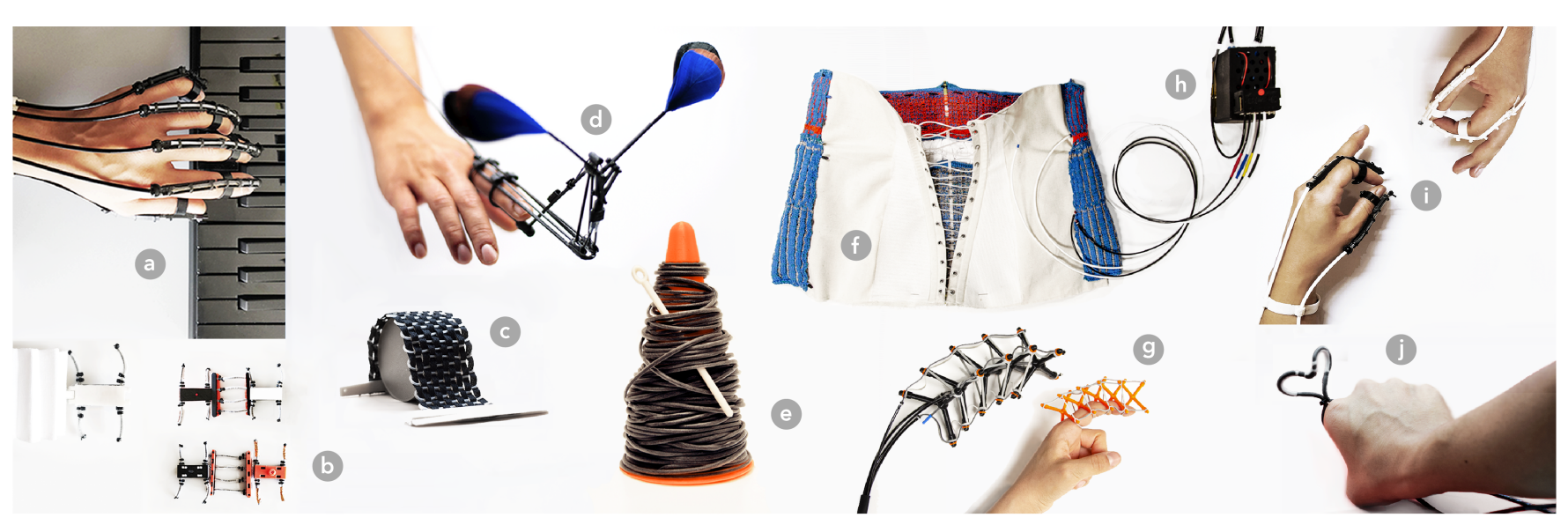

Figure 1: OmniFiber in use: (a) An assistive fexoskeleton for piano learning. (b) Patchbot. (c) A woven swatch with OmniFiber. (d) Finger-worn kinetic jewelry. (e) 30 meter OmniFiber spool. (f) Interactive corset inlay-knitted with OmniFiber. (g) Synchronized spine tensegrities with active OmniFiber tendons. (h) FlowIO hardware. (i) Wearable sign language. (j) Heart-shaped dynamic ring.

\begin{abstract}
Fiber - a primitive yet ubiquitous form of material - intertwines with our bodies and surroundings, from constructing our fibrous muscles that enable our movement, to forming fabrics that intimately interface with our skin. In soft robotics and advanced materials science research, actuated fibers are gaining interest as thin, flexible materials that can morph in response to external stimuli. In this paper, we build on fluidic artificial muscles research to develop OmniFiber - a soft, line-based material system for designing movement-based interactions. We devised actuated thin $\varnothing_{\text {outer }}$ $<1.8 \mathrm{~mm}$ ) fluidic fibers with integrated soft sensors that exhibit perceivably strong forces, up to $19 \mathrm{~N}$ at $0.5 \mathrm{MPa}$, and a high speed of linear actuation peaking at $150 \mathrm{~mm} / \mathrm{s}$. These allow to flexibly weave them into everyday tangible interactions; including on-body haptic

Permission to make digital or hard copies of all or part of this work for personal or classroom use is granted without fee provided that copies are not made or distributed for profit or commercial advantage and that copies bear this notice and the full citation on the first page. Copyrights for components of this work owned by others than ACM must be honored. Abstracting with credit is permitted. To copy otherwise, or republish, to post on servers or to redistribute to lists, requires prior specific permission and/or a fee. Request permissions from permissions@acm.org.

UIST'21, October 10-14, 2021, Virtual Event, USA

(C) 2021 Association for Computing Machinery.

ACM ISBN 978-1-4503-8635-7/21/10 . \$ \$15.00

https://doi.org/10.1145/3472749.3474802
\end{abstract}

devices for embodied learning, synchronized tangible interfaces for remote communication, and robotic crafting for expressivity. The design of such interactive capabilities is supported by OmniFiber's design space, accessible fabrication pipeline, and a fluidic $\mathrm{I} / \mathrm{O}$ control system to bring omni-functional fluidic fibers to the HCI toolbox of interactive morphing materials.

\section{CCS CONCEPTS}

- Human-centered computing $\rightarrow$ User interface toolkits.

\section{KEYWORDS}

soft actuator, stretchable sensor, microfluidics, e-textiles, haptics, movement-based HCI

\section{ACM Reference Format:}

Ozgun Kilic Afsar, Ali Shtarbanov, Hila Mor, Ken Nakagaki, Jack Forman, Karen Modrei, Seung Hee Jeong Klas Hjort Kristina Höök, and Hiroshi Ishii. 2021. OmniFiber: Integrated Fluidic Fiber Actuators for Weaving Movement based Interactions into the 'Fabric of Everyday Life'. In The 34th Annual ACM Symposium on User Interface Software and Technology (UIST '21), October 10-14, 2021, Virtual Event, USA. ACM, New York, NY, USA, 17 pages. https: //doi.org/10.1145/3472749.3474802 


\section{INTRODUCTION}

Thin linear forms are plain yet ubiquitous geometric building blocks found throughout nature. For example, most of peripheral neural cells are linear, branching out through the different parts of our bodies essential for our kinesthetic perception. Similarly, human skeletal muscle tissues are structured of bundles of fibrous muscle modules which help navigate our bodily movements and everyday voluntary control. When it comes to everyday objects and wearables, we spend most of our lives in direct contact with fibers and yarns. Such linear forms are versatile and their affordances allow them to be structured into textiles, that are in close correspondence with our bodies and movements.

Artificial fiber-based actuators (e.g. yarn/string/thread actuators), pioneered within the robotics community [17, 31, 36, 60, 61, 66, 67] are attracting increasing interest as a means to achieve seamless and high-granularity actuated on-body wearables and textile systems. In this paper, we introduce OmniFiber, a reconfigurable material system based on interactive fluidic fiber ${ }^{1}$ actuators (Figure 1).

Omnifiber goes beyond previous work in fluidic actuators by considering the needs and constraints of interaction designers, rooted in its design criteria, defined as 'weavability,' 'accessibility,' and 'actuation versatility' (Figure 2a). For the fabrication, we developed an accessible pipeline that can be performed with off the shelf materials. This fabrication technique embeds the sensing component into the fiber, allowing to use the strain tunability of the fibers to achieve a wide range of movement-based I/O. For output, Omnifiber supports actuation versatility in terms of strength (weak to strong), speed (immediate to ambient), shape (straight to complex linear geometries) as in Figure 2b, and modularity. Omnifiber is also skin safe, and compatible with common textile processing techniques -sewing, weaving, and machine knitting- due to its thin, pliable form and suitable surface friction. To support dissemination of the technique, we performed a preliminary characterization of the actuator elongation, bending and force output. This was used to create a minimalist mathematical model to aid designers' actuator selection. The overarching goal of this work is to create a powerful platform for actuating fibers that empowers interaction designers and Human-Computer Interaction (HCI) researchers.

While a variety of such line-based tools for on-body and movement-based HCI research have been already explored, they had major drawbacks to be woven into the fabric of everyday life [76]. Due to their actuation methods, they have been either rigid and bulky [41, 43], exhibiting slow response speeds with a limited movement range [14], provide low actuation forces and relatively small usable strain while being unsuitable for direct skin contact $[18,38,40]$. OmniFiber combines multimodal haptic feedback such as lateral skin stretch, compression, textured, and high frequency vibration feedback within a single thin and compliant fluidic fiber system, allowing for immediate response (axial displacement peaking at $150 \mathrm{~mm} / \mathrm{s}$ ), high strain (up to $245 \%$ ) and high force output (up to $19 \mathrm{~N}$ ).

To support designers, we outline a twofold design space; A) the primitive functionalities for designing morphing interfaces and movement-based interactions with OmniFiber, and B) 2D and 3D

\footnotetext{
${ }^{1}$ In this paper, we use a less restrictive definition of fiber, as a flexible cylindrical body with high aspect ratio and a diameter in the range of hundreds of microns.
}

fiber compositions utilizing modularity and traditional fiber structuring techniques to demonstrate complex morphing and combined haptic feedback. For control, we utilize and expand upon FlowIO [63], a compact and portable pneumatic control platform, to avoid large air compressor systems that often limit mobility, and allow users to easily orchestrate the interactivity of OmniFiber-based devices on-the-fly. With our relatively accessible implementation, we envision our work to be adopted by a variety of interaction designers in the e-textiles community, as well as practitioners and academics in movement-based [22] somaesthetic [24], or wearable interactions.
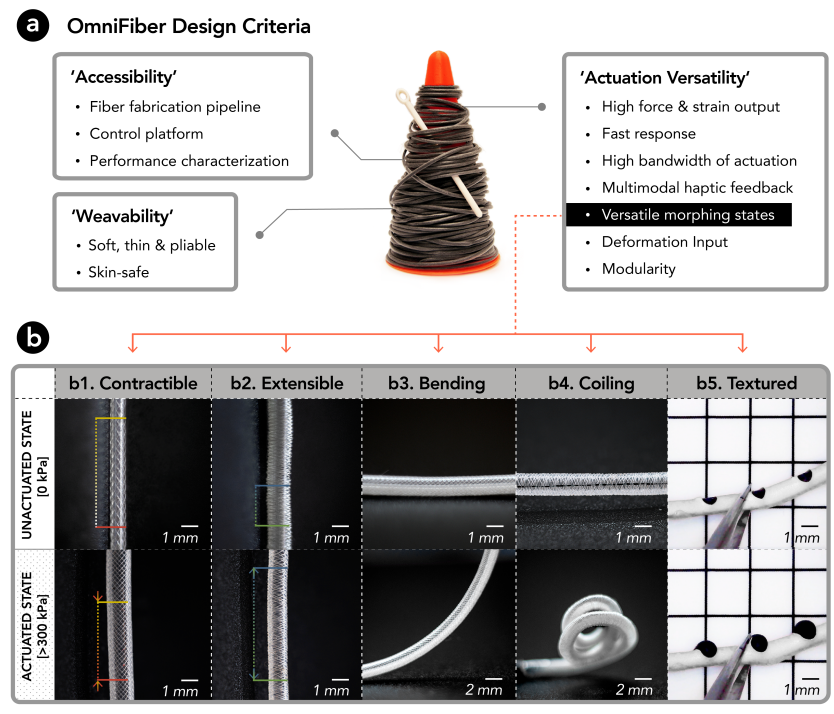

Figure 2: OmniFiber's (a) Design Criteria and (b) versatile morphing states as a guide for interaction designers: b1) contraction (by $32 \%$ ), b2) extension (by 245\%), b3) bending, b4) coiling, b5) textured.

In summary, OmniFiber's key contribution lies in the merging of components and simultaneous properties, into a comprehensive fiber-based system that:

- combines closed-loop strain control, shape programmability, modularity, and miniaturization for seamless textile integration in a single package. These are popular affordances in $\mathrm{HCI}$ actuators research, but are novel for thin McKibbens;

- presents an integrated fabrication pipeline for fiberreinforced fluidic actuators that provides affordances of value to designing on-body interactions [49];

- opens a design space that covers single fiber, 2D and 3D configurations;

- implements a FlowIO-based [63] control system with HW/SW add-ons, including high current circuit, high flow rate valve configuration, MUX sensor circuit, and Arduino library for multichannel strain control.

While each of these properties may not be novel in itself, together they make OmniFiber uniquely fitted to the needs of interaction designers as will be demonstrated through a range of design cases, such as on-body haptic devices to support movement-based skill acquisition, synchronized tangible interfaces for remote communication, and robotic crafting for expressivity. To the design 
community, the functionality and aesthetics of the material is not measured solely in terms of what it looks like as it moves, but more importantly, what it feels like interacting with our skin, movements, and bodily engagements. Our vision for OmniFiber is to provide the experience of a second muscle that interacts with our dexterous muscles, enriching physical interactions.

\section{RELATED WORK}

Research in shape-changing and actuated Tangible User Interfaces (TUIs) have been explored for dynamic affordances, physical displays and movement-based interactions [10, 25, 59]. OmniFiber contributes to several streams of research in this effort -fluidic interfaces, reconfigurable modular toolkits, and soft fiber-based actuator- to support movement-based interactions.

\subsection{Fluidic Interfaces}

In the past decade, soft interfaces utilizing fluid pressure for actuation have gained interest within the HCI field as organic, compliant, and lightweight interfaces for visual and haptic feedback $[12,19,35,39,46,47,50,70,73,78,85]$. In the field of soft robotics, pneumatic actuators are some of the most prevalent actuators due to their high power density, large deformations, accessible fabrication with of-the-shelf materials, and high bandwidth of actuation. These benefits have facilitated their dissemination to HCI for applications including haptic sleeves, full-body suits, and thin film shape displays [12, 35, 85]. Among them, PneuSleeve [85] is one of the most relevant works to OmniFiber, where researchers introduced a pneumatic forearm sleeve with a wide range of haptic expressions. The main difference is that OmniFiber is a deformation sensor integrated fiber-form actuator, and can be knitted and woven into a rich swatch of fabric patterns resulting in unique actuation modalities including out-of-plane bending, coiling and tactile micro textures. OmniFiber can also be sewn into other sheet substrates, while PneuSleeve is an already-integrated sheet interface. Another advantage of using multiple fiber actuators over a single sheet-form actuator is that if one or more of the fibers malfunction, the multi-fiber sheet assembly still continues to work with little leakage.

\subsection{Reconfigurable Modular Toolkits and Morphing UIs for Movement-based Design}

A variety of hardware tools with/for morphing UIs have been proposed in the HCI field [20, 41, 43, 44, 46, 57, 65, 65, 84]. In many of these works, researchers have integrated motion sensing and actuation capability within one module, to propose a way that allows users to program the motion by direct manipulation with their hands. This method of physical motion design is defined as 'kinetic memory' [57] or 'programming by demonstration [11] / example [32]' in HCI / HRI.

Inspired by this research, we developed OmniFiber to be coupled with deformation sensing to allow kinetic memory function in addition to the software GUI of motion control. For example, in MorphIO [46] researchers introduced a modular pneumatic I/O hardware kit based on tentacle-like actuators embedded with an elastic sponge for strain sensing. Compared to MorphIO, OmniFiber offers similar I/O capability in a form factor that is over ten times thinner (0.6 - $1.8 \mathrm{~mm}$ versus $1.6 \mathrm{~cm}$ in diameter) and with much higher resolution and versatility of a single module movement. Other motion design toolkits introduced fiber-form factors with reconfigurability using SMAs $[49,53,74,84]$. While these SMA actuators are often thinner than OmniFiber, they rely on sensing with an additional hardware which sacrifices the thinness of the fiber. Our technique with airbrushing of soft stretchable conductive traces on the actuator substrate helps maintain the thinness while allowing deformation input at once.

\subsection{Soft Fiber-based Actuators in HCI}

Soft fiber actuators have emerged as an area of interest in HCI as a means to package the rich interactivity of morphing line-based UIs $[14,40,43,75]$ in a lightweight and easily embedded (e.g. by sewing, knitting, weaving) manner. Among them, shape memory alloys (SMAs) are one of the most popular types adopted by HCI researchers for tactile interfaces, hair displays, paper craft, and curved surface prototyping [18, 48, 49, 51, 84]. While commonly used, SMAs have numerous drawbacks such as being potentially harmful to the human skin by overheating [13], requirement for a restorative force, arduous training process for reversible actuation [26], and being either too stiff (thicker SMAs) or too delicate (thinner SMAs) which in turn affects the actuation speed [3]. Furthermore, they are characterized as 'not easy to control' in [55], exhibiting low accuracy, thermal hysteresis and very low efficiency.

Twisted and coiled actuators also yield low efficiencies due to thermal processes underlying actuation that leads to excessive heating and rapid drainage of batteries when larger forces are needed [82]. Furthermore, inefficient passive cooling makes motion control slow and difficult [37]. Nevertheless, such actuators may have good use for passive actuation, where there is large variation in ambient temperature. In ModiFiber [14], the authors coated such twisted and coiled actuators in silicone, allowing for two-way morphing, but with no programmable out-of-plane behaviour. Although this actuator exhibits fast torsional actuation $(\approx 6 \mathrm{~s})$, its linear actuation speed is limited to $5 \%$ in $5 \mathrm{~min}$, while extensible-type OmniFiber's peak linear actuation speed is $70 \%$ in $\approx 1 \mathrm{~s}$. ModiFiber also has relatively small usable strain (5\%), and overheats during actuation $\left(80^{\circ} \mathrm{C}\right)$ which prevents direct body contact. Additionally, for both SMAs and ModiFiber, power must be continuously supplied to keep the fibers actuated, resulting in lower power efficiency compared to OmniFiber which can maintain its actuated state without drawing any power.

Albaugh et. al [1] introduced a compelling method for embedding cable tendons $[4,72]$ into fabric by computer-controlled weft knitting, allowing a variety of morphing behaviors that require motor control for interactivity, and reliable machine-knitting of delicate conductive yarns for embedded sensing.

While such parallel research in HCI enables novel interactions using the form-factor of a single line, the integration of dynamic fluid properties to a thin, composite fiber geometry remains unexplored. In the 2017 review paper, Designing Line-Based Shape-Changing Interfaces by Nakagaki et al. [42], it was discussed how the fluidactuated approach was mainly limited in size, mobility, and shape complexity. OmniFiber closes this gap by being developed with very thin, meters-long hollow elastic fibers $\left(300 \mu \mathrm{m}<\varnothing_{\text {inner }}<760\right.$ $\mu \mathrm{m})$ actuated by high pressure fluidics. While our design system is based on thin McKibben muscles [30] previous work is limited 
to only simple axial contraction capability of the muscle fibers. These devices are actuation-focused and do not include closed-loop feedback control. [61] Furthermore, machine-knitting of higher hierarchical structures with thin McKibbens -likely incompatible thickness with standard knitting machines- or ModiFiber -due to limited continuous fiber length- have not been implemented.

\section{A. PRIMITIVE FIBER ARCHITECTURE}

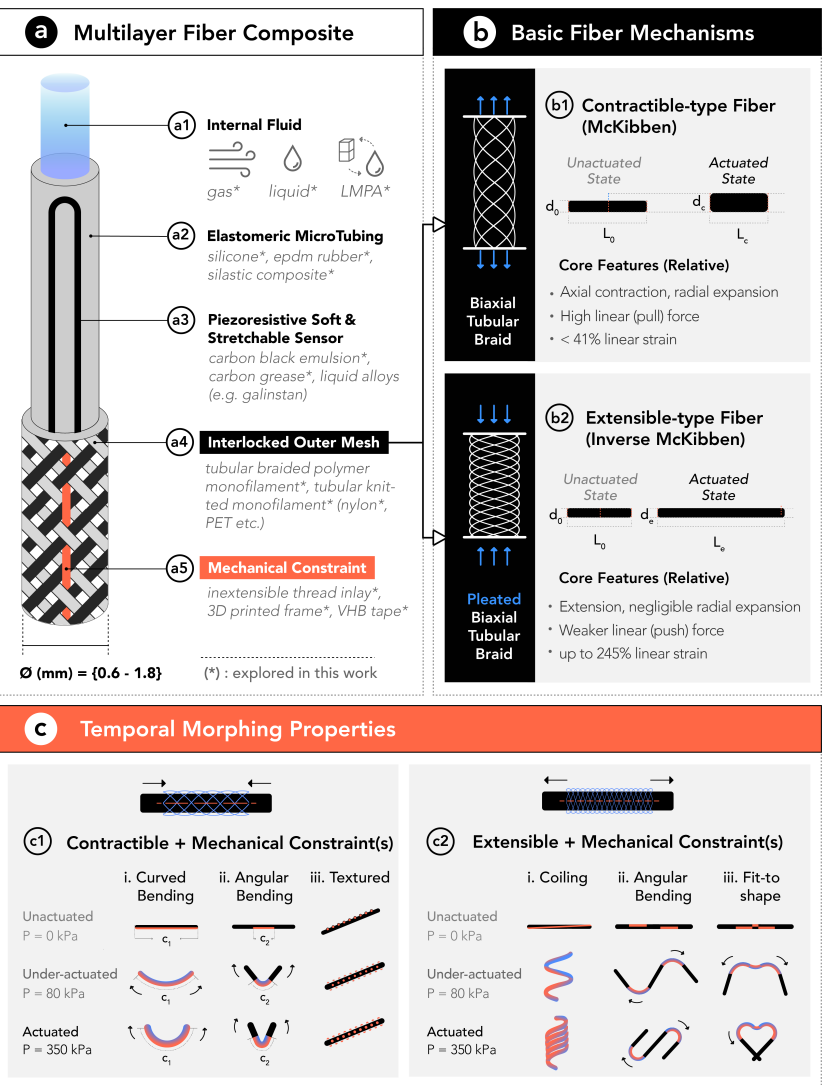

d) Multimodal Haptic Properties

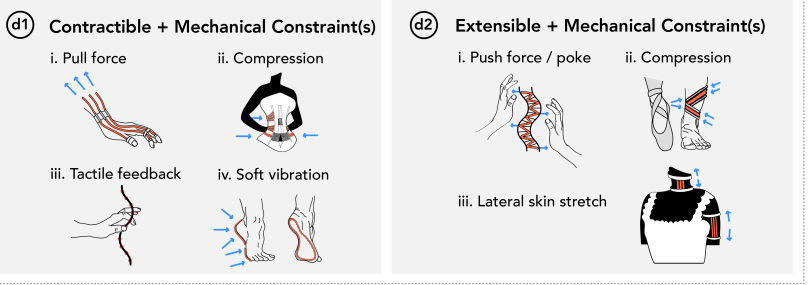

Figure 3: Section A: (a) Multi-layered fiber composite. (b) Two basic fiber working mechanisms. (c) Temporal morphing properties. (d) Multimodal haptic properties.

\section{OMNIFIBER DESIGN SPACE}

OmniFiber is a reconfigurable material system for movement-based interaction design, based on thin fluidic fiber actuators with closedloop strain control. We explore how I/O capabilities and multimodal haptic feedback of thin fluidic fibers can be leveraged to develop novel tangible interactions.
We define a twofold design space of OmniFiber as the fundamental architecture of fluidic fiber actuators, in which we describe how it can be composed for reconfigurable interactive functionalities. The design space consists of two main components; (A) Primitive Fiber Architecture, and (B) Modular Compositions and Fiber Structuring Techniques.

\section{1 (A) Primitive Fiber Architecture}

This section presents a hierarchical architecture of the fiber anatomy. We illustrate our multi-layered fiber (Figure 4a-b) that can exhibit one of the two types of basic mechanisms, and extending to a versatile set of morphing properties.

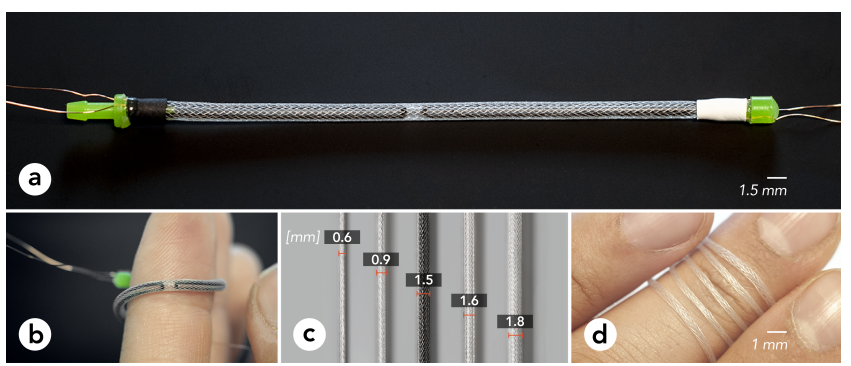

Figure 4: Primitive Fiber Architecture: (a) A multi-layered, $\varnothing$ $=1.5 \mathrm{~mm}$ OmniFiber composite. (b) around the index finger. (c) various diameter OmniFiber devices with varying tubing - braid properties (Supplementary Material 1). (d) $ø=0.6 \mathrm{~mm}$ fiber wrapped around the finger.

(a) Multilayer Fiber Composite: We devised five construction primitives for the fabrication of the multilayered OmniFiber design with a diverse set of properties (Figure 3a). These properties allow for a range of design choices to achieve the primitive fiber motions. (Figure $3 b$ ) Varying the parameters of the latter two construction primitives, namely the (a4) Interlocked Outer Mesh and (a5) Mechanical Constraint, effectively alters the morphing behaviour and haptic feedback properties as illustrated in Figure 3c-d.

a1. Internal Fluid is the medium contained in the fiber that dynamically flows when pressure is applied or removed. The internal fluid can be pressurized gas (i.e. air), liquid (i.e. water), conductive liquid metal, phase-changing low-melting point alloy (i.e. Gallium) or other specialized fluids such as a ferromagnetic fluid. The reported systems that use liquids vary in operating pressures and actuation methods. [71, 77] For hydraulic operation, noisy and miniaturized diaphragm pumps can be replaced by piezoelectric micro pumps, thus making compact design possible for wearability comfort.

a2. Elastomeric Micro Tubing. In this study, we use ethylene propylene diene monomer (EPDM) rubber and silicone elastomer micro-core tubing with properties detailed in Supplementary Material 1. For applications requiring higher force outputs, tubing materials with high elastic modulus, such as soft EPDM rubber, are preferred. There is a variety of commercially available tubings (Figure $4 \mathrm{c}-\mathrm{d}$ ) with outer diameters below $2 \mathrm{~mm}$ and lengths up to $100 \mathrm{~m}$ long. The internal structure of the tubing used in this study is cylindrical single lumen ${ }^{2}$ with a minimum diameter of $300 \mu \mathrm{m}$.

${ }^{2}$ The cavity or channel within a tube. 
a3. Soft and Stretchable Sensor. Sensing of user input is crucial for interactive applications which is why we designed a special sensor with a thin, tubular form-factor. We devised an accessible process using airbrush with conductive solution to fabricate highly stretchable $(130 \%)$ and thin $(100 \mu \mathrm{m})$ sensor channels that accommodate strains experienced by the human skin (55 to 75\%) [62]. We used resistive sensing, where mechanical strain causes a change in electrical resistance, to detect deformation inputs like stretch and pressure for closed-loop control.

a4. Interlocked Outer Mesh. Although this work mainly focuses on the effect of outer mesh interlocking technique on the actuation behaviour, it is also possible to utilize a wide diversity of yarns with varying mechanical properties, such as inherent yarn elasticity (detailed in Section 4.2). We detail the design and fabrication of two types of interlocked tubular outer mesh: braided (Figure 5a) and knitted (Figure 5b).

Braided mesh is the traditionally used method for sleeving McKibben actuators, where the actuation behaviour is affected by several parameters of the mesh such as braiding material, single strand length in the braid, braiding angle, braid diameter and expansion ratio. We studied a range of these parameters and their effect on the fiber actuation detailed in Supplementary Material 1.

Further, we devised an automated method to fabricate a theoretically infinite (in our case 60 meters) length strand of OmniFiber by applying a tubular knitted mesh around the elastomeric tubing using the circular mode of our weft knitting machine as will be detailed in Section 4.2. Although we achieved only $12 \%$ contraction ratio with the knitted sheath, while still higher than NiTi (2-5\%) and ModiFiber (5\%), a 2016 work on knitted-sheath McKibbens [5] demonstrates up to $50 \%$ contraction which is far greater than what standard McKibben actuators $(<40 \%)$ can achieve. Still, it should be noted when choosing an interlocking method, that knitted sleeving may be prone to higher hysteresis due to greater potential effect from friction between the sheath and elastomeric tubing.

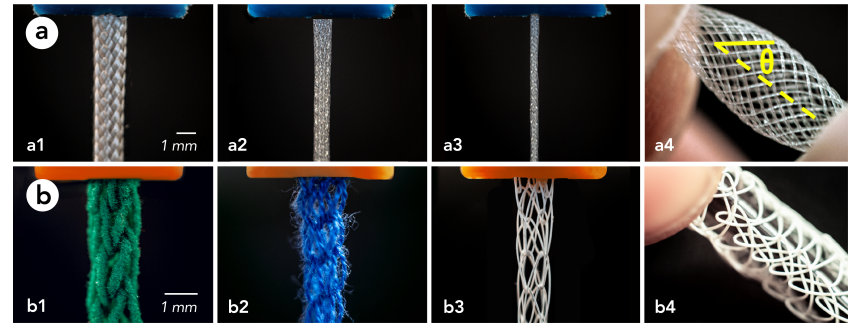

Figure 5: Macro view of interlocked outer mesh prototypes. (a) Off-the-shelf braided mesh: a1) copper silver, $\varnothing=1.6 \mathrm{~mm}$ ), a2) nylon, $\varnothing=0.8 \mathrm{~mm}$, a3) nylon, $\varnothing=0.4 \mathrm{~mm}$, a4) expanded diameter at braid angle $\theta=54.4^{\circ}$. (b) Machine-knitted tubular mesh: b1) elastic yarn, b2) cotton yarn, b3) polyester yarn, ø $=1.2 \mathrm{~mm}$, b4) expanded diameter.

(b) Basic Fiber Mechanisms: OmniFiber primitives are based on one of the two primitive fluid-driven muscle fibers; Contractible Muscle Fiber (McKibben) and its inverse counterpart Extensible Muscle Fiber. The operation principle of both fibersis similar by nature; the inner elastomeric microtubule deforms in axial and radial directions under applied pressure, and this leads to a force output and displacement in the axial direction by altering the braid angle. The Contractible Muscle Fiber shortens in length when fluid is supplied to the actuator $[64,81]$ and produces strong linear forces in the pull direction. In contrast, the Extensible Muscle Fiber extends in response to the internal fluid pressure.

The working principle of the basic primitives is characterized by the relationship between the internal tubing and the braided outer mesh. When the outer braiding angle, $\theta$ (Figure 5a4) is smaller than $54.4^{\circ}$, the fiber contracts, and when it is larger than $54.4^{\circ}$, it extends [33]. The extension and contraction limit of a fiber is determined by the filaments in the braid no longer being able to slip past each other beyond the $54.4^{\circ}$ angle state, as they are in direct contact. In order to design the primitive fiber motion, one can pick a fiber type to either prioritize force output or range of morphing behaviour.

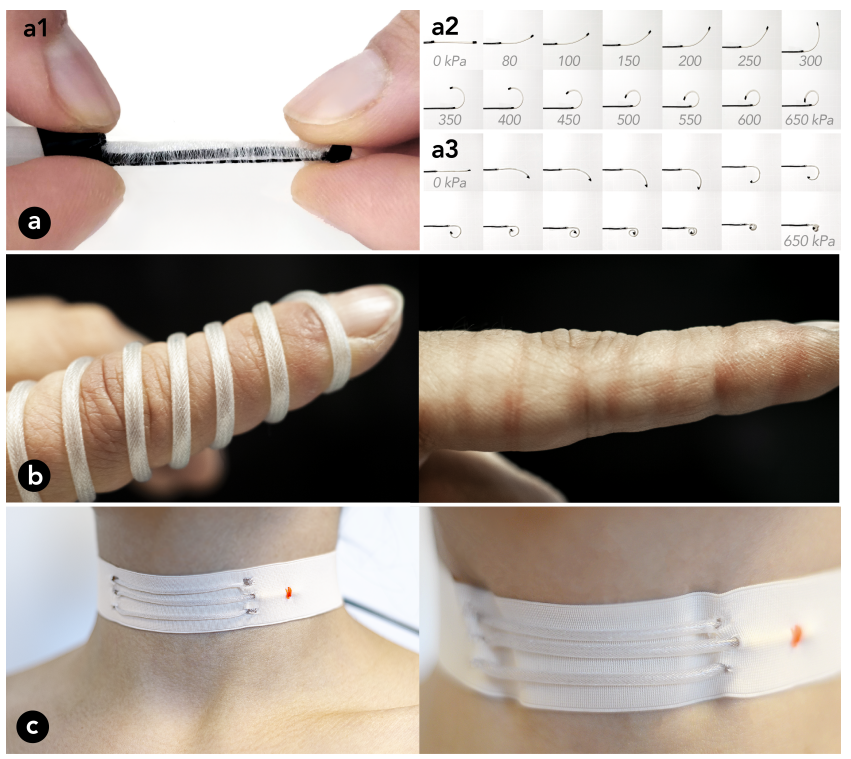

Figure 6: (a) Temporal morphing properties: a1) A strain limiting inextensible thread inserted parallel to the braided mesh axis for mechanical constraint. Temporal shapechange from line to varying bending radii curves for, a2) contractible-bending fiber, a3) extensible-coiling fiber at pressures between $0 \mathrm{kPa}$ and $650 \mathrm{kPa}$. Both fiber types are strain-limited with a single constraint along the actuator length. Multimodal haptic properties: (b) compression feedback with contractible OmniFiber on finger. (c) OmniFiber sewn into a fabric piece for lateral skin stretch and vibration feedback on the larynx.

a5. Mechanical Constraint. We extend the McKibben design's simple contraction and extension motions by adding mechanical constraints on the interlocked outer mesh. The basic motion of the fibers can be mechanically programmed to bend at a certain angle or to fit arbitrary linear shapes. Mechanical Constraints are either added via an inlaid inelastic thread on the outer mesh (Figure 6a1), or by a $3 \mathrm{D}$ printed flexible constraint placed on the actuator. To 
support the design of the constraint and the resulting curved shape, we developed an empirical model detailed in Section 6.2.

Based on this core architecture, the rest of the design space section A presents the extended design primitives in two categories: morphing and haptic properties.

(c) Morphing Properties: A wider space of expressive movement behaviours can be utilized for interaction design to dynamically represent information in a tangible way, such as sending tangible messages in morphing line forms, or for bottomup form-finding practice based on a line geometry for design applications.

By controlling the length, location, orientation, and number of mechanical constraint layers added on the basic OmniFiber anatomy, we can create a variety of geometrical shapes and movements in a similar vein to hydrostatic skeletons in nature [69] The fiber then can be bent, folded, or coiled in multiple directions in order to achieve reversible targeted shapes in 3D, from a single linear form. Each temporal state of the morphing fiber can be maintained by keeping the control valve at the off state. We captured the temporal morphing of a similar length of extensible (Figure 6a2) and contractible type (Figure 6a3) two basic fiber primitives, patterned with a mechanical constraint layer along the full length of the actuator. We observed behaviours between $0 \mathrm{kPa}$ and $600 \mathrm{kPa}$ at increments of $50 \mathrm{kPa}$ for both. The first perceivable shape deformation was observed at $80 \mathrm{kPa}$ for both primitives. While the extensible fiber coils at maximum actuation due to the strain percentage being higher $(245 \%)$ than that of the contractible fiber $(32 \%)$ which only goes as far as closing a full circular shape.

(d) Haptic Properties: As OmniFiber can be easily embedded into clothing, it makes a viable material for interaction designers to fabricate a variety of on-skin and on-body haptic sensations such as pulling, squeezing, poking, vibrating, and skin stretching (Figure 6b). Multiple haptic properties can be combined in a single OmniFiber device, such as on-finger compression feedback (Figure 6c) that can be actuated at high frequency to achieve vibration feedback simultaneously.

In addition to the macro deformations like bending and coiling, small scale $\left(\varnothing_{\text {outer }}=0.7 \mathrm{~mm}\right.$ ) morphing can be achieved such as a textured surface for tactile feedback. (Figure 2e) This can be fabricated by knitting the outer mesh with sub-millimeter lace stitch patterns that creates holes for which the inflated flexible tubing bulges through.

Section A of the design space describes the fundamental architecture to construct OmniFiber for embodying targeted shapes, tunable morphing, and haptic properties. In Section B (Figure 7) we present assembled, embedded or structured Omnifiber assemblies to achieve more complex interactivity. These multidimensional structures allow new utilities (e.g. out-of-plane haptic feedback), and to produce higher force (e.g. by weaving OmniFiber as weft) and strain (e.g. by inlay knitting OmniFiber) outputs.

\section{2 (B) Modular Compositions and Fiber Structuring Techniques}

This section presents two OmniFiber configuration types: (a) assembling multiple fibers in modular and reconfigurable compositions

\section{B. MODULAR COMPOSITIONS \& FIBER STRUCTURING TECHNIQUES}

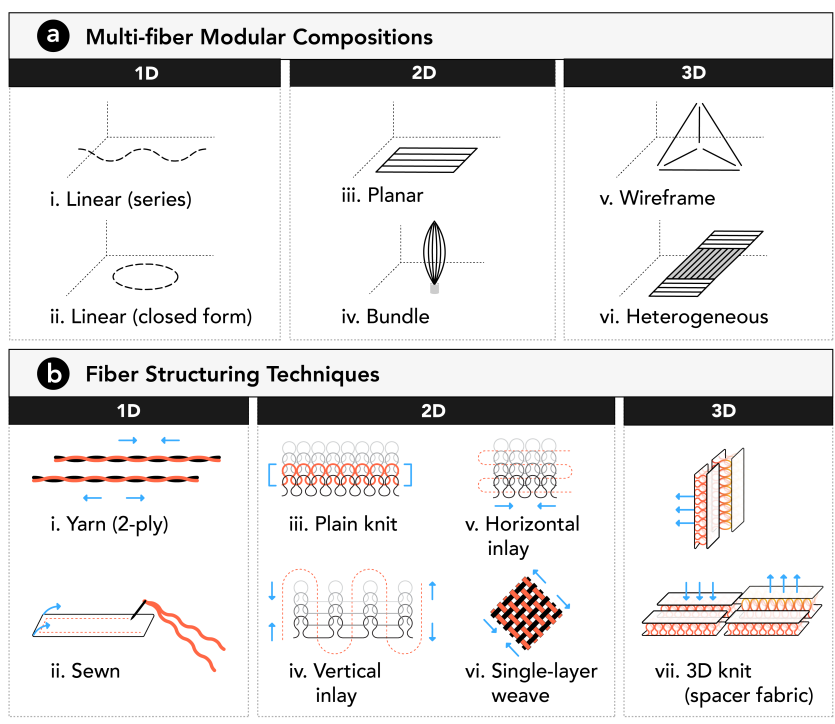

Figure 7: Section B: (a) Modular fiber compositions. (b) Fiber structuring techniques.

(Figure 7a) and (b) the structuring a single continuous fiber into fabric assemblies (Figure 7b).

(a) Multi-fiber Modular Compositions. The first type of composition is the use of multiple fibers in $1 \mathrm{D}, 2 \mathrm{D}$, and 3D compositions (Figure 8a-c). While a single OmniFiber is relatively easy to fabricate, it has certain limitations (e.g. insufficient forces on certain body parts, relatively limited number of movement types). As a design guideline, to obtain larger forces one can freely increase the number of actuators in parallel or bundles [30]. Researchers have also shown that it is possible to achieve higher strain outputs by braiding the fibers in hierarchical configurations [29]. Furthermore, the fibers can be arrayed in various configurations to achieve omnidirectional actuation (Figure 7a:i-iv). An additional level of modularity can be achieved by composing reconfigurable multifiber modules. Such heterogeneous composition (Figure 7a:vi) can
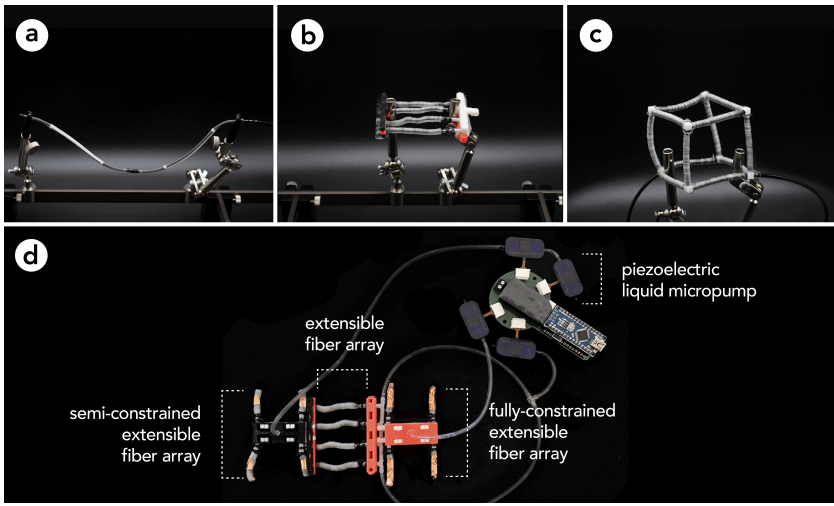

Figure 8: Modular fiber compositions: (a) 1D linear. (b) 2D planar. (c) 3D wireframe. (d) heterogeneous assembly. 

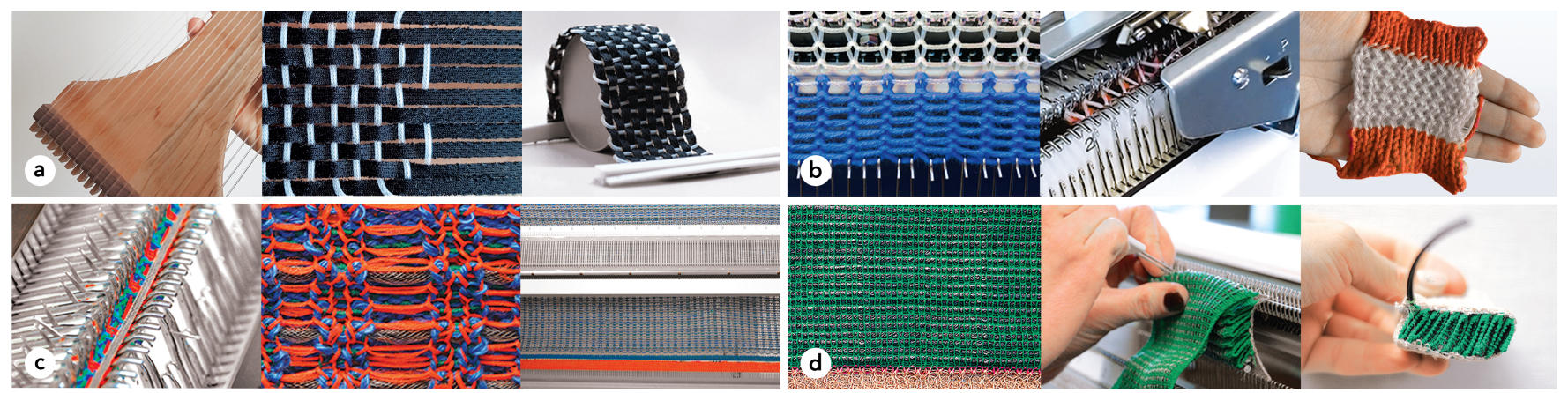

Figure 9: OmniFiber-based swatches with various fiber structuring techniques: (a) Woven with a continuous $ø=0.9 \mathrm{~mm}$ OmniFiber as weft. (b) Plain knitted and rib knitted $\varnothing=0.9 \mathrm{~mm}$ OmniFiber. (c) Inlay-knitted large fabric piece using $30 \mathrm{~meter}$ long $\varpi=1.5 \mathrm{~mm}$ OmniFiber. (d) Weft-knitted quasi-spacer fabric with active $ø=1.5 \mathbf{~ m m}$ OmniFiber spacer layer.

involve hybrid types of fiber modules, (e.g. extensible, bending, helical) in one larger assembly (Figure 8d).

Morphing and Haptic Properties. When multiple of the same functional fibers are arrayed (e.g. a planar array of extensible fibers) one can create in-plane bending motions by selectively actuating (e.g. $350 \mathrm{kPa}$ ) or under-actuating (e.g. $80 \mathrm{kPa}$ ) individual fibers. In a higher level composition with heterogeneous fiber groups, one can achieve simple locomotion and morphing in 3D by sequentially actuating different muscle fiber groups, as demonstrated by the 'Patch-bots' kit in Applications 5.3.

A significant mechanical advantage of multi-fiber compositions is the relatively higher output force production compared to a single fiber composition. For example, while a single fiber provides enough force to bend the human finger, multi-fiber compositions can support the movement of larger body parts [29].

(b) Fiber Structuring Techniques. While a single fiber is limited to in-plane haptic feedback (e.g. compression, skin stretch), a 3D quasi-spacer fabric can exhibit out-of-plane haptic feedback pushing orthogonally towards the body. We present fiber compositions all of which are fabricated with traditional textile processing techniques on a $4.5 \mathrm{~mm}$ standard gauge flatbed weft knitting machine (Silver Reed SK840 + SRP60 ribber). By using long (>10 m) active fibers $\left(\varnothing_{\text {outer }}=0.9 \mathrm{~mm}\right.$ ) we exemplify weaving, plain, rib and inlay knitting (Figure 9a-c). As OmniFiber's polyester outer mesh provides suitable friction for machine-knitting, these techniques intend to demonstrate the scalability of OmniFiber to higher hierarchical structures.

Morphing and Haptic Properties. We explored different techniques to embed OmniFiber into a knitted structure and examined the resulting characteristics knit structures affect on the movement of the fiber and vice versa. The knit contains OmniFiber, that is inlaid within the fabric, therefore its constructed by two classes of fibers: one building the base structure and the other being activated.

In Figure 9b, we demonstrate a rib knitted swatch constructed entirely of $\varnothing=0.9 \mathrm{~mm}$ OmniFiber (with orange waste yarn), that changes stiffness when pressurized. Such variable stiffness fabric [9] can also be controlled locally by assembling separately knitted samples that together form a fully functioning wearable that partially stiffens when necessary and softens when mobility is required. Alternatively, one can use a phase-changing working liquid $[6,52]$ such as Gallium or other low-melting-point alloy (Figure $10 \mathrm{~d}$ ), actuated at liquid state using a piezoelectric liquid micro pump $(200 \mathrm{~L} / \mathrm{h})$ as in Figure 8d, and shape-lock in 2 hours at slightly lower than room temperature $\left(20^{\circ} \mathrm{C}\right)$. This property can be utilized more efficiently with an active cooling system.

Figure $9 \mathrm{c}$ demonstrates a large scale machine-knitted functional fabric by horizontally inlaying OmniFiber between the two beds at every other course in a serpentine trajectory. Additionally, we devised a method to fabricate a 3D quasi-spacer fabric (Figure 9d) on the same weft knitting machine by pleating-and-knitting the inlay-knitted fabric in place (Supplementary Material 6). The active 'spacer' layer is entirely made of a contractible-type OmniFiber, resulting in out-of-plane haptic feedback once actuated (Figure 10f). As 2D fabrics are often limited to in-plane feedback such as stretching, our 3D knitting technique allows a novel out-of plane feedback mechanism in textile structures. This opens a new set of haptic properties for actuated textiles, enabling design with force feedback orthogonal to the body.

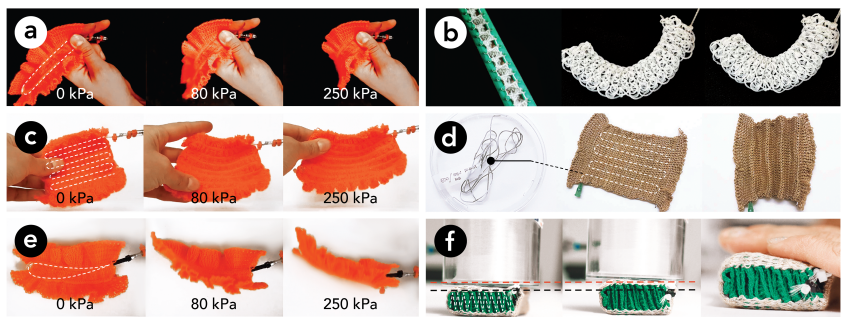

Figure 10: A rich in-fabric actuation palette demonstrating states from unactuated to actuated. (a) inlay-knitted contractible-bending swatch. (b) stiffness changing handknitted collar. (c) inlay-knitted extensible swatch. (d) inlayknitted Gallium-filled fiber for shape locking. (e) inlayknitted contractible-twisting swatch. (f) 3D knitted quasispacer for out-of-plane feedback. 


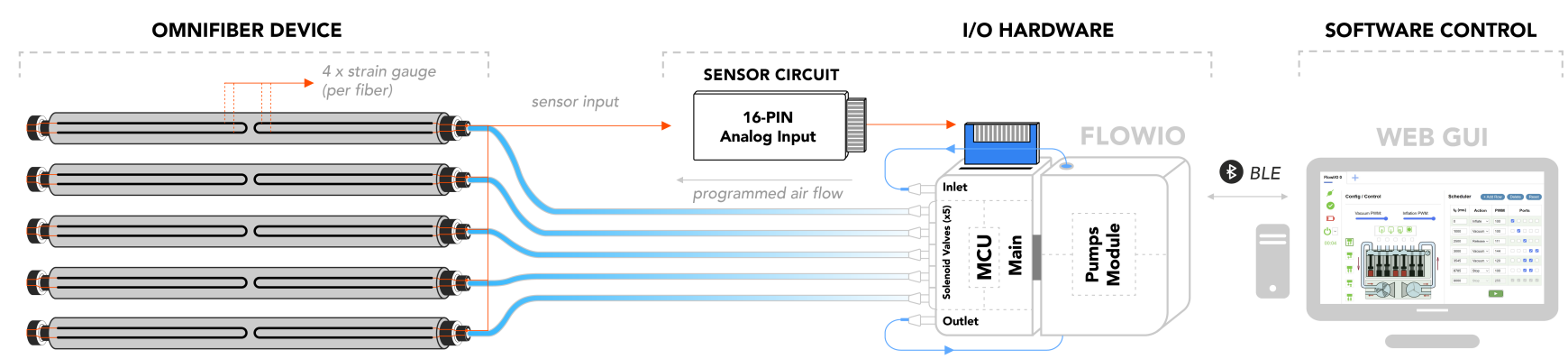

Figure 11: System architecture diagram.

\section{OMNIFIBER IMPLEMENTATION}

\subsection{System Architecture}

The overall design of our system consists of the following components: a flexible and stretchable fiber-based modular interface, a pneumatic development platform FlowIO, with web-based GUI, and a multi-channel, multiplexed analog input for closed-loop strain control (Figure 11).

Our system control is built on FlowIO [63], a miniature integrated fluidics control platform with five pneumatic ports. We extended the FlowIO hardware and software to fit the following requirements of our system: (1) yielding high pressures (up to $350 \mathrm{kPa}$ ), (2) an auxiliary multichannel sensor circuit for closed-loop strain control, (3) allowing real-time or planned "task scheduler" on the Web GUI, (4) communication between multiple devices for bidirectional interaction (Supplementary Material 4). In FlowIO, we use a serial inflation configuration of two miniaturized diaphragm pumps (Sparkfun, Airpo D2028 Vacuum Pump) yielding higher pressures to actuate multiple long $(\approx 1 \mathrm{~m})$ fibers at once. For a fiber to maintain its actuated state, power doesn't need to be continuously supplied as the valves have a normally-closed state when off. The Web GUI simultaneously connects via Web-BLE to multiple FlowIO devices, and is able to communicate bidirectionally. We use the FlowIO web app running in Chrome to receive the multichannel strain data from the 16-pin analog input module based on tangible deformation input by an OmniFiber device, process it in real-time, and control the output pressure (in the same OmniFiber device, or a paired second device) based on this data.

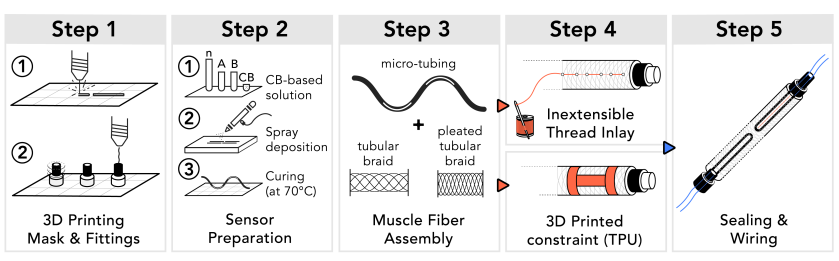

Figure 12: Multi-step fabrication pipeline of OmniFiber.

\subsection{Multilayer Fabrication Pipeline}

In this section, we overview the design and fabrication pipeline for OmniFiber (Figure 12). The fabrication pipeline starts with relatively accessible methods to ensure replicability of material science outputs by designers, such as the fabrication of stretchable sensor channels. This is followed by the assembly of the fiber composite with the outer mesh, adding mechanical constraints, and finalized with manual sealing, and wiring of the fiber for control.

Step 1: 3D Printing Mask and Pneumatic Adapters. To apply multi-channel conductive traces on the tubing, we 3D printed a 2-part mask from with ABS filament. The mask design has $4 \mathrm{U}-$ shaped channels (Figure 13a1), allowing for the wiring to be fixed at the fiber ends, as well as indents for the pneumatic adapters to be tightly fitted during spraying. Finally, prior to sensor fabrication, we inserted the 3D printed pneumatic adapters on both ends of the tubing (Supplementary Material 3).

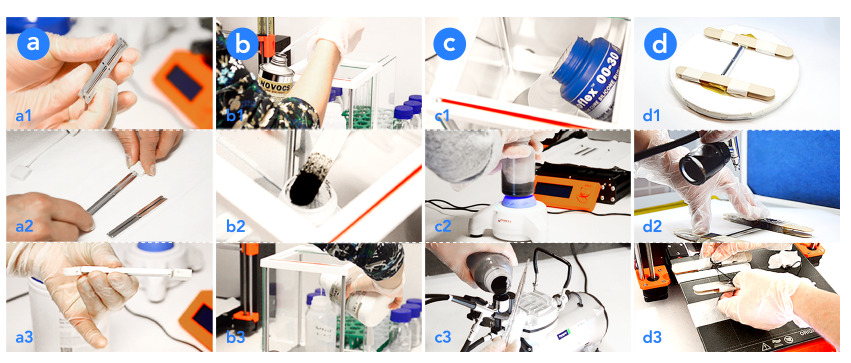

Figure 13: Step 2: Multi-step CB-based stretchable sensor fabrication. (a) Mask preparation: placing the tubing in the mask and clamping with screws. (b) CB powder-silicone solution preparation. (c) Mixing and pouring for spraying. (d) Applying airbrush at $15 \mathrm{~cm}$ distance from the mask, and curing on $3 \mathrm{D}$ printer hot bed.

Step 2: Sensor Preparation. We employed a spray deposition method of a carbon black nanopowders-elastomer composite to pattern multi-channel strain sensors on the elastomeric micro-tubing. Our custom designed mask allows fabricating $0.1 \mathrm{~mm}$ wide conductive traces on the tubular surface. The following multi-step sensor fabrication process (Figure 13) can be adopted for a larger number of channels as well as more complex geometries.

A sprayable solution was prepared by mixing $\mathrm{CB}$ nanopowders (Ketjenblack EC-300J, HM ROYAL) and elastomer liquids (Ecoflex 00-30, Smooth-on, Inc.) with a solvent (Novocs Gloss, Smooth-on, Inc.) to decrease the viscosity of the $\mathrm{CB}$-elastomer mixture. The $\mathrm{CB}$ powder $(0.15 \mathrm{~g})$ was first sonicated (RS PRO Ultrasonic Cleaner) in 
Novocs solvent (15 g) for 15 minutes (Figure 13b). Then, Ecoflex part A (1.5 g) was added and mixed manually for 5 minutes. Finally, Ecoflex part B (1.5 g) was added to the solution and mixed on a mini vortex mixer (Four E's) for one minute (Figure 13c). Before spraying, 3D printed tubing connectors were inserted into both ends of the tubing. A 3D printed mask covered the tubing while spraying with an airbrush at 30 psi for 25 iterations to achieve the starting resistance of $1 \mathrm{MOhm}$ for each sensor channel (Figure 13d). After curing the sprayed sensor patterns for 20 minutes on the 3D printer's hot bed (or one hour at ambient temperature), we consecutively sprayed two layers of Ecoflex part A + Novocs and Ecoflex part B + Novocs solutions as a protective coating layer for the sensor channels.

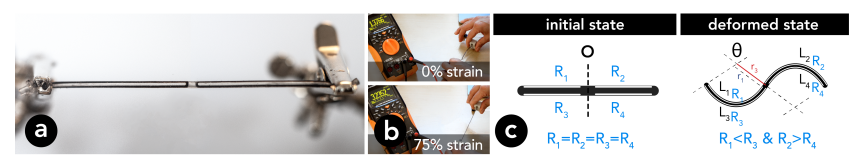

Figure 14: (a) 4-channel CB-based resistive strain sensor. (b) resistance values as $1,3 \mathrm{MOhm}$ at $0 \%$ and $3,2 \mathrm{MOhm}$ at $75 \%$ strain. (c) bending curvature principle diagram for an example double-bend deformation.

With this method, we patterned 4-channel strain gauge on each fiber module (Figure 14a) to allow for detecting radii and direction of curvature by comparing the strain-resistance values across reciprocal electrodes (Figure 14b-c). While our native approach leverages resistive sensing, the embedded fluid pressure sensor in FlowIO can also be utilized for closed-loop feedback control.

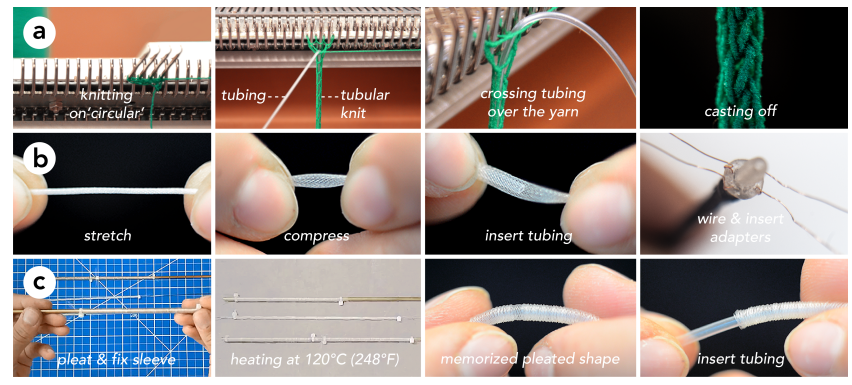

Figure 15: Step 3: Basic fiber assembly. (a) Knitting tubular mesh around elastomeric tubing on a weft-knitting machine for scaled fiber fabrication. (b) Contractible-type fiber fabrication. (c) Extensible-type fiber fabrication.

Step 3: Basic Fiber Assembly. Once the sensor channels are completely cured, we insert the tubing inside the tubular mesh.

Meshing with tubular knit. We devised an automated method to fabricate a theoretically infinite (in our case 60 meters) length strand of OmniFiber by knitting a tubular mesh around the elastomeric tubing on a single bed of our weft knitting machine (Supplementary Material 6). Such interlocking technique allows a wider design space by varying the properties of the tubular knit (e.g. diameter, stitch size and yarn characteristics) to program OmniFiber's movement in a single-step fabrication (Figure 15a).

Meshing with tubular braid. We used a commercially available braided nylon monofilament (Durometer 50A) with; diameters of 0.8 and $1.6 \mathrm{~mm}$, initial braiding angle of $18^{\circ}$, and respective braid counts of 24 and 32.

a. Contractible-type Fiber. The contractible-type fiber can be fabricated at any length, by inserting a similar length of elastomeric tubing in a tubular braided mesh of braiding angle smaller than $54.4^{\circ}$ (Figure 15b).

b. Extensible-type Fiber. The extensible fiber consists of identical materials as the contractible fiber, except the mesh should be considerably longer ( $x 4$ in our example). To achieve larger strains, the tubular braid is compressed into pleats using the following steps in Figure 15c. First, we insert a metal rod of the expanded diameter into the braided mesh as a mandrel. Then, we compress the mesh in the axial direction to create even pleats and fix both ends with a heat resistant nylon cable tie to maintain the pleated length. Finally, we heat the mesh either using a heat gun or oven for the structure to memorize the pleated shape, at a temperature lower than the melting point of the mesh material. Since the mesh is axially much longer, this allows the inner tubing to elongate until the braid angle reaches the $54.4^{\circ}$ locking angle state. With this composition, we achieved strains from $70 \%$ up to a maximum of $245 \%$.

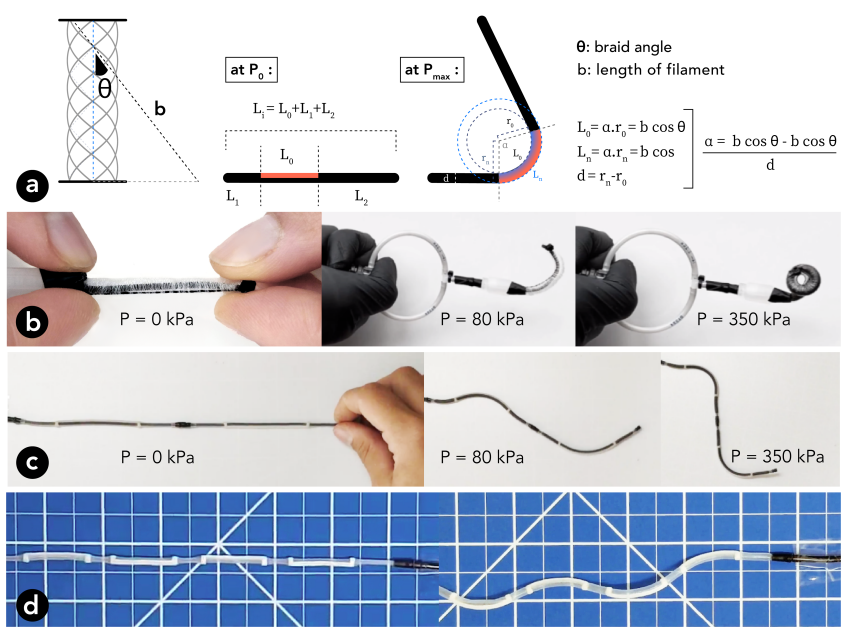

Figure 16: Step 4: Adding mechanical constraint. (a) bending curvature model based on braiding angle as a variable. (b) inextensible thread insert on extensible fiber. (c) 3D printed constraint on contractible fiber, a 1D modular assembly of two OmniFiber devices. (d) multiple 3D printed constraints on contractible fiber for multi-bend morphing.

Step 4: Adding Mechanical Constraint. We present two techniques of selective strain-limiting to mechanically program the morphing behaviour via; 1) a fixed inextensible thread insert (Figure 16b), and 2) a 3D-printed flexible constraint (Figure 16c).

Depending on the fiber mechanism type, the bending occurs in the direction of the constraint (extensible type) or opposite to it (contractible type). The length of the strain limiting segment 

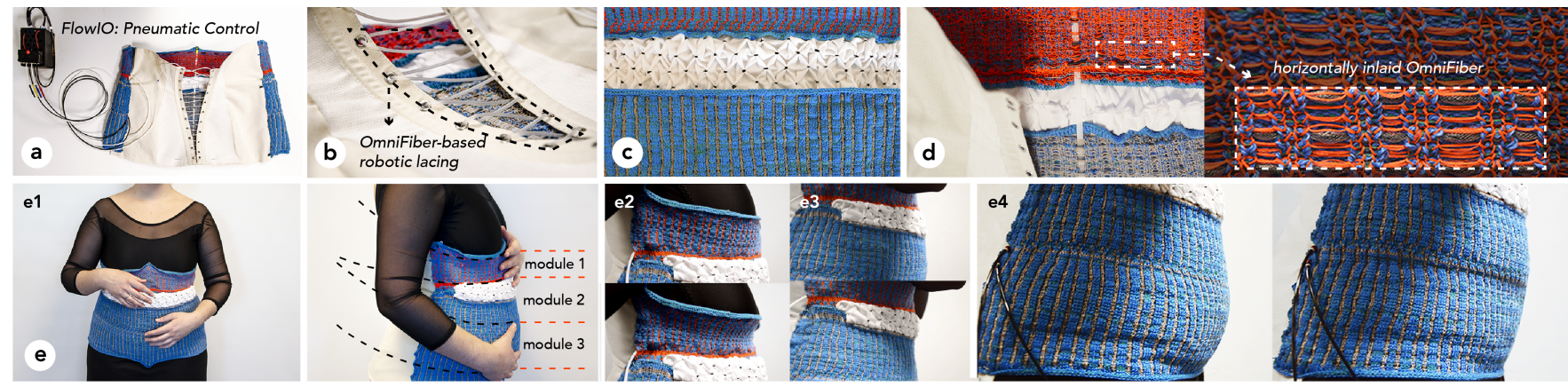

Figure 17: (a) Upper body garment for breathing guidance during singing, peristaltically controlled by FlowIO. (b) active corset lacing with OmniFiber. (c) corset front view with vertically inlaid fibers. (d) Horizontal inlay of OmniFiber and close-up view. (e) Modular corset on a professional singer's torso: e1) front and side view, and unactuated - actuated compression states of e2) module 1, e3) module 2, e4) module 3.

affects the bending radius of the actuator when actuated (Figure 16a), while its position affects the bending direction with resulting in-plane, out-of-plane or multi-plane bending motions.

(1) Inextensible Thread Insert. In this technique, we manually insert and fix an inextensible monofilament parallel to the braided mesh axis (Figure 16b). This causes the local mesh geometry where the insert is located to be constrained in strain for both contraction and elongation-type mechanisms. We used a low elastic modulus, cheap nylon monofilament (fishing line) to bypass the impact of stretchability of the monofilament on the resulting movement. The thickness $(0.05 \mathrm{~mm})$ and material of the filament chosen should be similar to the strands in the braided outer mesh to avoid potential failure such as a ripped mesh.

(2) $3 D$ Printed Constraint. The strain limiting section can be fabricated by $3 \mathrm{D}$ printing a thin flexible frame. We printed multiple linear constraints $(0.2 \mathrm{~mm}$ thick, $0.8 \mathrm{~mm}$ wide) dispersed along the contractible type fiber with a $\emptyset_{\text {outer }}=1 \mathrm{~mm}$. We used TPU-type non-brittle filaments on a low-cost FDM 3D printer (Figure 16d). The printed part is then inserted between the braided outer mesh and the tubing, in parallel to the fiber axis.

Step 5: Wiring and Sealing. Surface-insulated copper wires (38 AWG, 3522, Adafruit) are connected to the sensor electrodes on both ends of the fiber with a carbon conductive ink (Carbon Conductive Adhesive 502, EMS). To achieve stability at the connections we used silicone glue (DOWSIL 732, Dow Corning). Finally, for a robust end sealing and further avoid fluid leakage, we inserted a flexible miniature Polyolefin Plastic heat-shrink tubing (McMaster Carr) at the tube-fitting connection points.

\subsection{Closed-loop Strain Control and Web-GUI}

As a first step, to achieve a simple strain mirroring, we start with the known relationships between the actuator $\Delta$ length vs applied pressure, and actuator $\Delta$ length vs $\Delta$ resistance. From these relationships, we devised a mathematical mapping between the strain-induced resistance change and pressure (Supplementary Material 5). When the user tangibly deforms (e.g. stretch) an input OmniFiber device (1), the strain sensors change their resistance, from which we compute the deformed state. We then utilized our mapping algorithm between strain and pressure to determine what pressures need to be supplied to the output OmniFiber device (2) to exhibit a similar deformation behaviour (e.g. elongation) and vice versa.
To obtain the resistance of the strain gauges in real-time, we use a 16-channel analog input expansion module of FlowIO [63], which contains multiplexers and voltage dividers with resistance values tailored specifically for OmniFiber's resistance profile (1 Mohm to $17 \mathrm{Mohm})$. The strain sensor data is sampled continuously and a moving average is computed over the last 10 samples from each channel. The averaged data is then continuously sent via Bluetooth Low Energy (BLE) to a central device running the FlowIO App. The FlowIO App simultaneously connects to the two FlowIO devices via BLE. After it receives the strain data from an input FlowIO device (1), it processes according to our mapping algorithm between strain and pressure, and then actuates the corresponding pneumatic port of the output FlowIO device (2) to supply the pressure for achieving the desired motion.

\section{APPLICATIONS}

In this section, we demonstrate how the design space of OmniFiber can be used for functional interactive applications. We envision OmniFiber-based interfaces as on-skin, on-body and on-object applications, woven into our daily environments.

\subsection{OmniFiber Interfaces for Movement Learning Support}

We explored how OmniFiber can be used to embody 'somatic tacit knowledge' [21] in creative movement practices. With these applications, we envision OmniFiber as a tangible tool to support and augment the expressive somatic vocabulary of the user.

Upper body garment as diaphragm breathing guidance for vocal training. Controlled breathing is fundamental to professional singing: muscle movements in the singer's torso allows for sensing of the different breathing patterns while singing. Such need for communicating the felt, non-verbal experience of breathing methods between a vocal trainer and trainee is highly relevant, both for in-person and remote settings.

Working closely with a classically trained singer, we designed an interactive singing corset (Figure 17a) as a haptic guide to grasp the felt experience of diaphragm breathing for a novice vocal trainee. The garment design consists of three modules that simulate a peristaltic breathing movement on the wearer's body (Figure 17e) by a sequential pressurize $(450 \mathrm{kPa})$ and exhaust $(0 \mathrm{kPa})$ task scheduling. 
For each module, we used $\varnothing=1.5 \mathrm{~mm}, 10 \mathrm{~m}$ long contractible-type fibers with high elastic modulus EPDM tubing to achieve localized compression feedback by horizontal inlaying (Figure 17d), and lateral skin stretch by vertically inlaying OmniFibers (Figure 17c). Uniform compression pressures of $1.2-6.5 \mathrm{kPa}$ is achieved, allowing a range for light to stronger compression on the torso [56]. Furthermore, the garment's fit can be dynamically customized to the wearer's body by actuating a single continuous OmniFiber lacing of $\varnothing=1.8 \mathrm{~mm}$ at the back of the corset (Figure 17b).

Asynchronous Exo-muscles to Support Learning Musical Expression. During the training process on a musical instrument for novice players, the tangible haptic guidance of an exo-muscle device can allow the practice of polyrhythmic ${ }^{3}$ musical expression We designed a system controlled by two FlowIO devices (Figure $18 \mathrm{a}, \mathrm{b})$, programmed to purposefully disrupt the synchrony between the two hands of the player (Figure 18b).

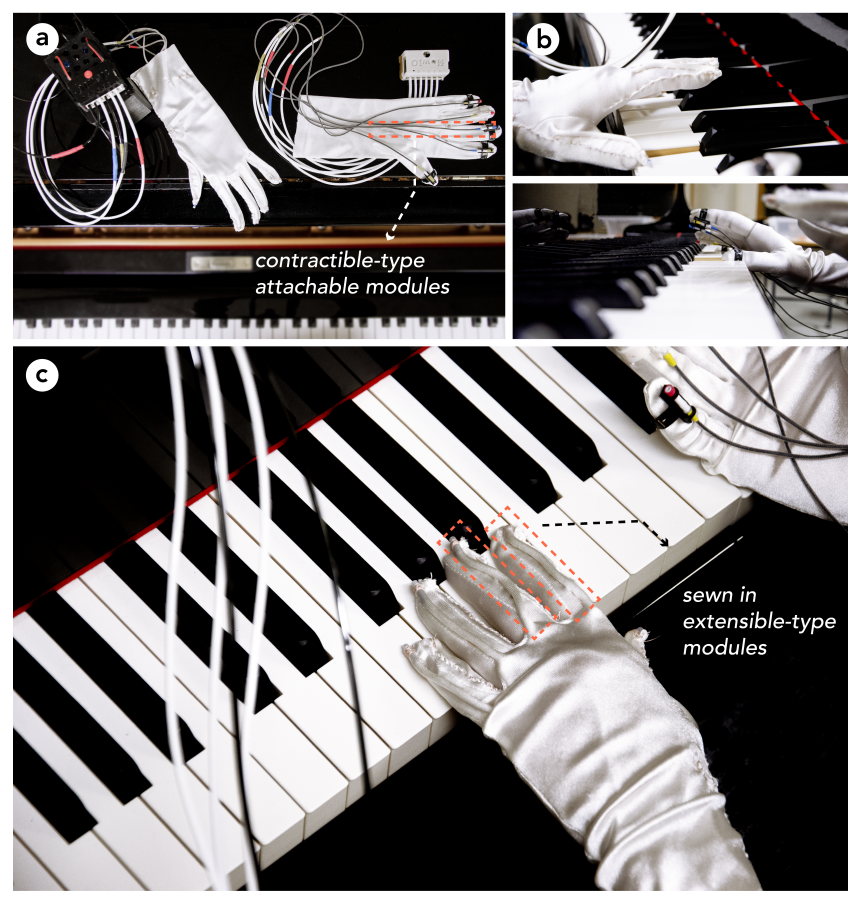

Figure 18: (a) OmniFiber-embedded "skill gloves" for practicing polyrhythmic expression in piano, controlled by two FlowIO devices that communicate via BLE. (b) Two hands playing different beat rhythms. (c) OmniFiber seamlessly sewn into the performance glove.

While previous research explored soft exoskeletons using pneumatic actuators $[66,67,79]$, in this example we utilize a $\varnothing=1.5$ $\mathrm{mm}$ contractible fiber attached to the anterior (Figure 18a) of each finger in a tight-fitting glove, that produces a maximum tensile strength of $\approx 16 \mathrm{~N}$ at $600 \mathrm{kPa}$. The posterior of finger sections are also woven $\varnothing=2 \mathrm{~mm}$ extensible-type fibers (Figure 18c) to augment consecutive flexion-extension motion at switching frequencies of

${ }^{3}$ Polyrhythm is the simultaneous use of two or more rhythms that are not readily perceived as deriving from one another, or as simple manifestations of the same meter.
$10 \mathrm{~Hz}$ for each finger's keystroke, and generate forces up to $8 \mathrm{~N}$ at the fingertip [54].

OmniFiber-based Spine Tensegrity to Reflect Body Movement. Awareness to one's spine posture and movements during an activity - from static posture to dynamic dance practice - significantly affects one's movement skills. However, as the vertebrae are internal to the body, their posture during such activities is often covert.

We designed two devices; (1) An orange 'body worn tensegrity device' that functions as a deformation sensor-only, while the (2) black 'standalone tensegrity model' is actuated based on this sensor data mapped from the body worn device (Figure 19a). The body worn device consists of rigid 3D printed vertebrae, a laser-cut rubber lattice as a passive tendon, and five $\varnothing=0.9 \mathrm{~mm}$ strain sensing layer of OmniFiber to capture the multi-DoF deformation of the wearer's spine in real time. The standalone model has five individually controlled contractible-type $\varnothing=1.5 \mathrm{~mm}, 60 \mathrm{~cm}$ long OmniFiber modules with a maximum $27 \%$ contraction ratio, functioning as active tendons to playback the movements of the wearer's spine. We achieved a maximum displacement of $16 \mathrm{~cm}$ when all five modules are simultaneously actuated, sufficient for the displacement requirements of the human spine during a dance practice. We envision the tangible playback of the standalone model to aid in communicating small adjustments to a dancer's movement in learning contexts.

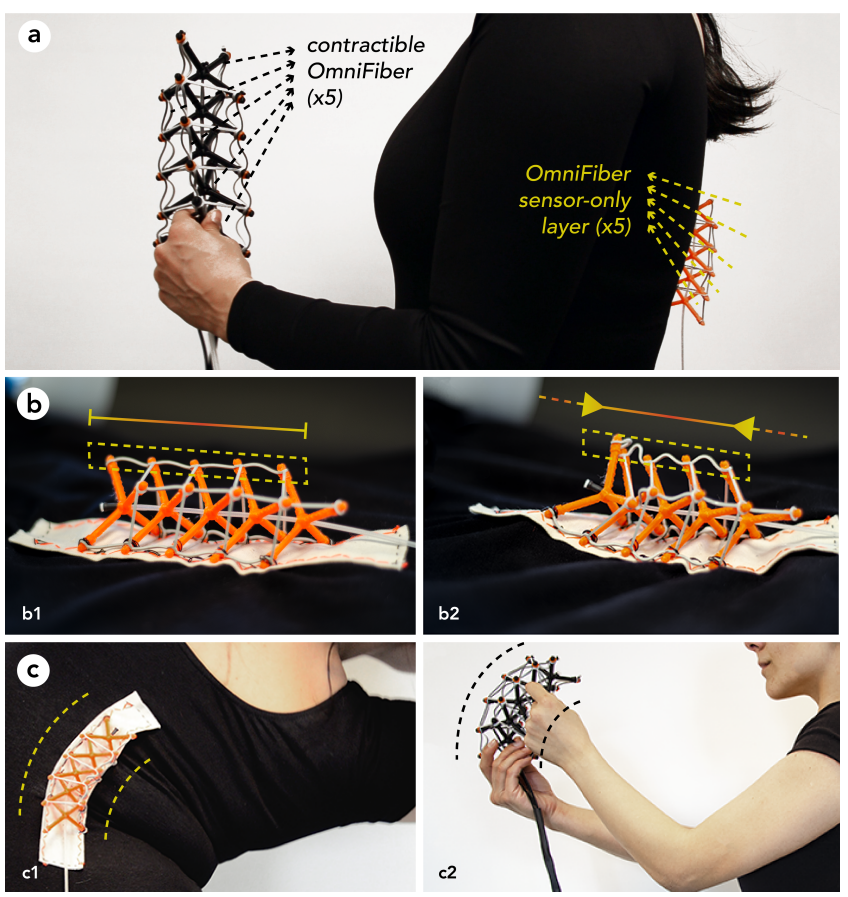

Figure 19: (a) Standalone model and body worn tensegrity design. (b) Body worn tensegrity on dancer's body: b1) undeformed and b2) deformed states. Tangible record \& playback: (c1) capturing spine movement with body worn tensegrity, (c2) replaying and observing on the standalone model. 


\subsection{Tangible Interfaces for Remote Communication}

Remote Communication by Tangible Sign Language. Researchers previously developed sign language to speech translation [83], however, while spoken language enables expression through rhythm and intonation, the rich tangible expressions in sign language computationally mediated by haptics remains underexplored within HCI research. OmniFiber devices attached onto users fingers can remotely mediate the sign-based communication by adding haptic feedback to it, leveraging dimensions such as intensity, pace and rhythm, as supporting haptic vocabulary (Figure 20a).

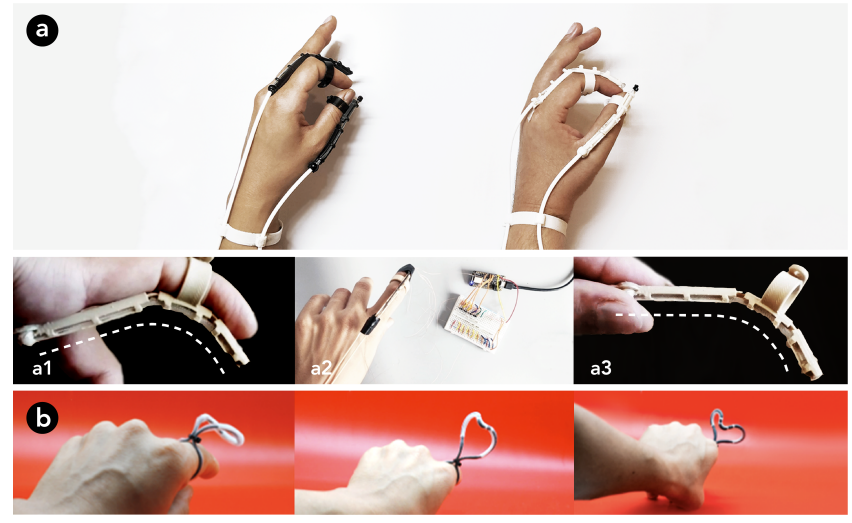

Figure 20: (a) Haptics-mediated sign language by record \& playback of finger motions. (b) An OmniFiber-based ring morphing into an iconic heart shape as a tangible message.

On-body Tangible Notifications. A wearable OmniFiber ring or bracelet can receive and convey information tangibly by dynamically moving and changing its shape. We implemented a linear ring made from a $\varnothing=1.5 \mathrm{~mm}$ contractible fiber with three mechanical constraints. The constraints are designed and located to morph the ring into an iconic heart shape, to notify when a message from a loved one is received (Figure 20b).

\subsection{Robotic Cables, Tendons and Threads}

Finger-worn Kinetic Jewelry. We demonstrate how on-body dynamic and expressive appearance can be achieved with OmniFiberactuated kinetic jewelry [27]. We designed a finger-worn device resembling a flapping magpie bird, actuated by iterative pressurerelease of a 2-ply extensible-type OmniFiber device. These thin devices functioning as active tendons, enable the integration of morphing materials into the delicate aesthetics of jewelry (Figure 21a).

Bio-inspired Robotics for Expression and Play. Inspired by natural structures that can perform complex movements [69], we fabricated a toy monkey with an active tail (Figure 21b1) made of an extensible strain-limited OmniFiber, demonstrating a playful coiling actuation (Figure 21b2). The tail is robust enough to pick up small objects (Figure 21b3) and carry the toy's own weight (Figure 21b4).
Movement Sequence Learning with Patch-bots kit. Utilizing multi-fiber configurations in our design space, we designed a modular kit of OmniFiber patches, which we call 'Patch-bots', capable of simple inchworm locomotion (Figure 21c). It consists of three modules; an array of extensible fibers forming the body, and two pairs of mechanically constrained extensible limbs for gripping capability. Users can learn assembly and locomotion control by sequentially actuating the separate modules connected to FlowIO, and using the WebGUI task scheduler to choreograph the locomotion (Figure 21c2).
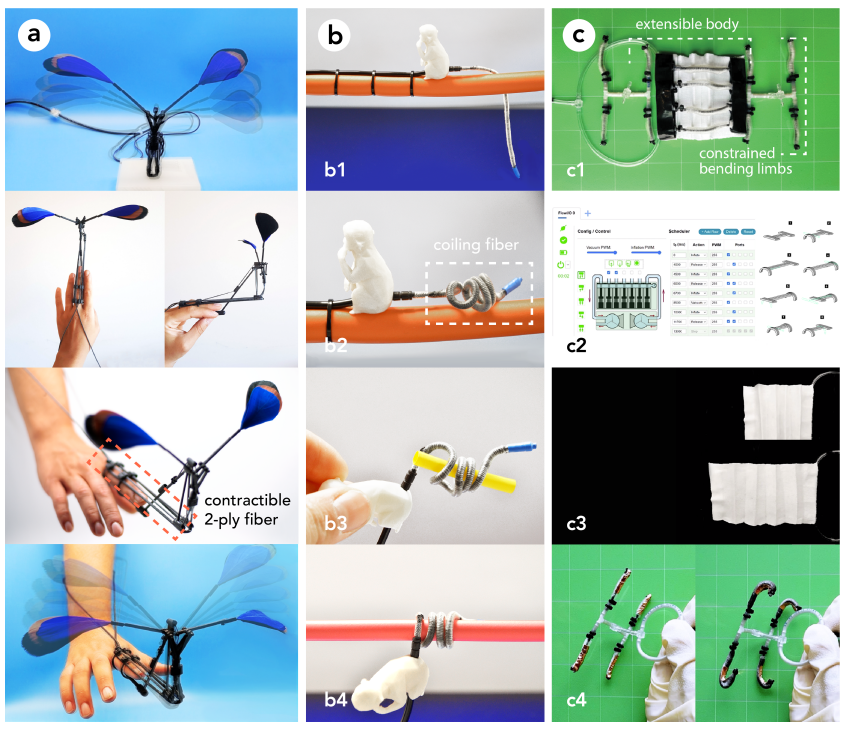

Figure 21: (a) Finger-worn expressive kinetic 'magpie' jewelry actuated by 2-ply contractible-type OmniFiber. (b) A toy monkey with a coiling type robotic tail that can b3) pick up or b4) wrap around objects. (c) Patchbot Kit, a heterogeneous modular assembly robot with $2 \mathrm{D}$ OmniFiber arrays that can: c3) extend its body and c4) bend its limbs for locomotion.

\section{TECHNICAL EVALUATION}

\subsection{Sensor Characterization}

Resistance changes of the fabricated sensor were monitored under tensile strains at $18 \mathrm{~mm} / \mathrm{sec}$. up to $130 \%$ strain by 10 iterations. As shown in (Figure 22a), a small hysteresis was observed, which is induced by percolation network changes of the conductive composites. The repeatability of resistance changes of the sensor was obtained.

\subsection{Actuator Mechanical Tests}

Based on our experiments, OmniFiber may be operated at varying pressures yielding different strain and force outputs. As contractibletype muscle fibers have already been characterized in detail by previous work [30], in this section we mainly present our experimental and analytical findings for displacement and geometry change in extensible-type fibers. 
For the elongation experiment setup, we used actuators made with silicone tubing ( $\varnothing=1.6 \mathrm{~mm}$, Silastic), 1/16" diameter braid with a braid count of 32 and initial braiding angle of $18^{\circ}$.

Axial Displacement Testing: Our aim in establishing a design space of thin fluidic fibers is to allow designers to have adequate flexibility to capture any desired behaviour for a variety of applications. Although we take the initial length of the actuator as the only variable, we provide a parsimonious model $^{4}$ based on our experimental data (Supplementary Material 2).

We ran axial experiments with four different lengths of extensible fibers; 50, 100, 150, and $200 \mathrm{~mm}$. In our setting, the fibers have three distinct actuation states (Figure 22b):

State 0: Air fills in the tubing (no geometric change, just volume) State 1: Unconstrained expansion and extension of the tubing until it touches the outer mesh radially (convex)

State 2: Constrained extension only - Now the tubing is constrained both radially and axially, which in turn results in change in the outer braid angle (down to $54.4^{\circ}$ balance point). In this state the tubing cannot expand radially anymore, thus only elongation is observed which has a concave behaviour.

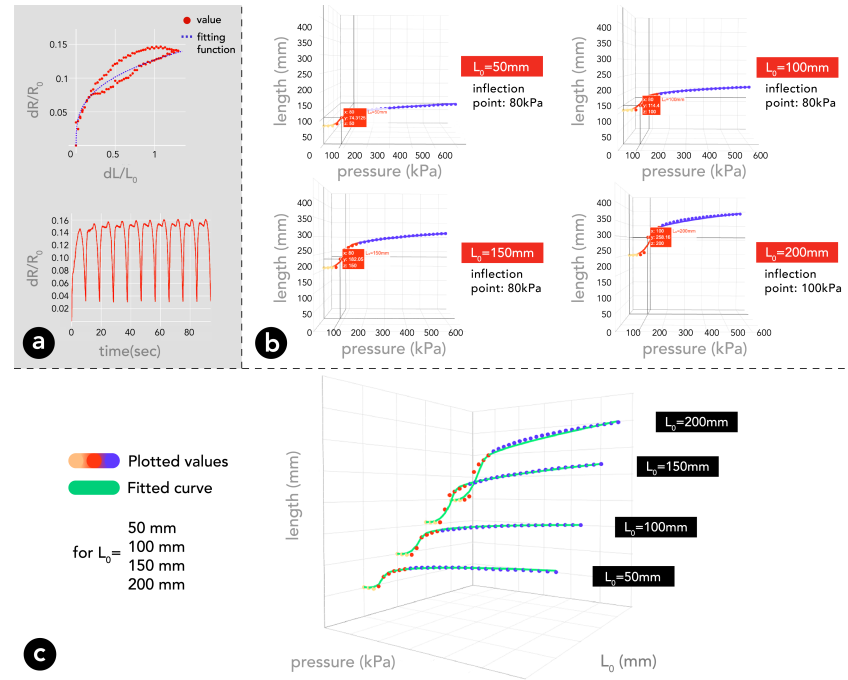

Figure 22: (a) Resistive sensor profile; resistance-strain and resistance-time. (b) Experimental data plotting for 4 different initial lengths of extensible muscle fiber: 50, 100, 150, 200 $\mathrm{mm}$. (c) Elongation model curve fitting for all initial lengths.

Empirical Elongation Model: As stated above, each fiber has two distinct actuated states (State $1 \& 2$ ) with different constraints on its behavior. Let's denote the actual behavior of the fiber in State 1 and State 2 by $f_{1}: P \times L \rightarrow L$ and $f_{2}: P \times L \rightarrow L$, respectively, where $P=[0,600]$ is the set of pressure levels, and $L=[0, \infty)$ is the set of length levels.

${ }^{4}$ Parsimonious models explain data with a minimum number of parameters, or predictor variables.
Below is our parsimonious model of the actual behavior, $\hat{f}: L \times$ $P \rightarrow L$, where each component of the piece-wise function corresponds to the respective states. Note that the model has no exogenous parameters, apart from the pressure and the initial length:

$\hat{f}\left(p ; l_{0}\right)= \begin{cases}l_{0}+5 \cdot 10^{-4} p^{2}+3 \cdot 10^{-5} p^{3} & p \in\left[0, \phi\left(l_{0}\right)\right) \\ f_{1}\left(\phi\left(l_{0}\right)\right)+2\left(\sqrt{p-\phi\left(l_{0}\right)}\right)+\gamma l_{0}\left(p-\phi\left(l_{0}\right)\right) & p \in\left[\phi\left(l_{0}\right), 600\right]\end{cases}$

where $\phi\left(l_{0}\right)=80+\frac{2}{15}\left(l_{0}-50\right)$ is the "state-changing" pressure in $\mathrm{kPa}$ for the fiber with initial length $l_{0}$, and thus, $f_{1}\left(\phi\left(l_{0}\right)\right)$ is the "statechanging" length of the fiber with the initial length $l_{0}$ under pressure $\phi\left(l_{0}\right)$, and $\gamma\left(l_{0}\right)=-0.05+1.8 \cdot 10^{-3} l_{0}+1.4 \cdot 10^{-4} l_{0}^{2}+2.66 \cdot 10^{-8} l_{0}^{3}$ is the coefficient of the linear term of the fiber in its second state with the initial length $l_{0}$.

Our model is plotted and compared with the empirical results as shown below, demonstrating a good match for all four lengths. (Figure 22c) While this is not a final generalized model, it is a relatively useful model that allows designers to get a general idea of the actuation behavior before physical fabrication. Currently, our empirical data only has the initial length and applied pressure as variables, although we believe that the current parsimonious model will accommodate additional variables without significant change to the model.

Radial Bending: We conducted bending tests to verify our assumption regarding the relationship between mechanical constraint parameters-bending radius and pressure-bending radius. Our overall assumption is based on the observation that the mechanical constraint, whether on a contractible or extensible fiber, will completely limit the movement of the applied part, and there will be no length change in the constrained area. This, in effect, causes a bending behaviour due to the resulting difference in length on reciprocal sides of the fiber upon pressurization.

As we already have the elongation model, using the length of an arc formula we can calculate the bending radii of the fiber, $\alpha$ at any given pressure.

$$
\alpha=\frac{L_{P}-L_{0}}{D}
$$

where $L_{P}$ is the length of the unconstrained, thus elongated, side at pressure $P, L_{0}$ is the length of the constrained side of the fiber, and $D$ is the diameter of the fiber. Moreover, the inner bending radius, the perpendicular distance between the center of bending and constrained side of the fiber, is given by $r_{0}=\frac{L_{0}}{\alpha}$, and likewise the outer bending radius by $r_{P}=\frac{L_{P}}{\alpha}$.

Axial Force Testing: The contraction force of a single fiber is dependent on the actuator diameter, and increases with pressure in an approximately linear relationship. Since our fluid-actuated fibers are significantly thinner than the previously reported thin McKibbens, we conducted a Contraction \% - Contraction Force, and Pressure - Contraction Force test with our contractible fiber actuators (Supplementary Material 2). For the test setup, we used an HLB manual test stand with and an analog force gauge (Baoshishan 100lb. Push-pull force gauge), an external compressor and pressure regulator to measure the force and amount of contraction. 
We varied the supplied pressure at increments of $100 \mathrm{kPa}$, from $100 \mathrm{kPa}$ up to $600 \mathrm{kPa}$, where the maximum contraction ratio obtained from the vertical displacement guide is $32 \%$ and force production under $600 \mathrm{kPa}$ is $8.8 \mathrm{~N}$ with the $\varnothing=0.9 \mathrm{~mm}, 53 \mathrm{~A}$ fiber configuration in Supplementary Material 1, index 16.

\section{LIMITATIONS AND DISCUSSION}

This section details limitations and future directions of OmniFiber.

\subsection{Automated Fabrication Pipeline}

In our current fabrication pipeline of a single OmniFiber, multiple steps rely on manual processes (i.e., assembly, wiring, and coating) that are intensive, although quite reliable. When scaling the fabrication to a large number of fibers, the process would benefit from further automatization. A promising technology for automating OmniFiber fabrication would be leveraging multi-material 3D printing, to fabricate a conductive core and the interlocked mesh layer controlling localized flexible properties, $[15,58]$. To fabricate longer strands of OmniFiber for textile applications, recent thermal fiber drawing methods can be leveraged [28].

\subsection{Toolkit Evaluation, Design and Simulation Tool}

Though we have not yet done any formal evaluations, we provided an OmniFiber toolkit to three professional textile designers to assess the prototyping processes of robotic textiles using OmniFiber, over the course of 4 weeks. Although working with fluidic fibers in their practice allowed new ways of thinking about movement and designing the garment-body interaction, the number of parameters to control in achieving a desired actuation showed that a simulation tool would be beneficial to support the design pipeline. For example, one participant discovered that the inherent properties of the filament used in the outer mesh and its relative elasticity to the mechanical constraint thread has significant effect on the fiber's morphing behaviour. Another participant reported that she found it hard to imagine how the fabric would move when the fiber was knitted in a specific knit pattern. These suggest an advanced computational tool would be beneficial such as [23], where the simulation of knitted and woven fabric forms combined with fluid dynamics are supported by the tool, for further accessibility of our work.

\subsection{Soft, Silent and Embedded Fluidic Control System}

While the miniaturization of state-of-the-art power and control platforms, like FlowIO, is promising, the noise and rigidity limits the compliance and comfort of the user interface. A future research direction we plan to pursue is employing advancements in soft and stretchable pump technologies for actuating soft muscles [8] Such devices allow for the integration of the fluid supply into the wearable system, however current challenges are safety and energy efficiency. This could alternatively be solved by building systems that harvest energy from human motion to acquire high pressure fluid power.

\subsection{Expanding the Basic Architecture and Fiber Properties}

There exists a rich set of opportunities by expanding the Multilayer Fiber Composite construction primitives in Figure 3a, including some future considerations and challenges.

Scaling. When considering scaling, the largest obstacle with smaller tubing is the maximum force it will provide. The strength of the tubing follows the inverse of the diameter, hence it is the maximum hydraulic pressure that will set the limit. Typically, for a fiber muscle at 5 bar and with $1.4 \mathrm{~mm}$ inner diameter, the maximum contraction force will be around 15-20 N. In addition, from the Hagen-Poiseuille equation ${ }^{5}$, the pressure drop increases with the power of two to a smaller hydraulic diameter if the flow velocity $(\mathrm{m} / \mathrm{s})$ is kept constant. This will limit the reaction time of eversmaller tubing. One advantage is that the fracture strength of the tubing will increase with smaller sizes, but sub-millimeter tubing still cannot take too high loads. As shown in our experiments, a 16-filament ( $0.05 \mathrm{~mm}$ thick) braided, $0.6 \mathrm{~mm}$ diameter tubing can take up to $1 \mathrm{~kg}$ of load.

Sensor. A main challenge with our prototypes is that the placement of sensors require reiteration of channel length and location for each application. To overcome such a challenge, future prototypes could benefit from an increased number of sensor channels along the actuator and machine learning to minimize accumulated errors from dense sensor readings $[7,16]$, or optimize the number of sensors for a given application [68].

Elastomeric tubing. Although not explored in this work, an exciting future design space is to customize the internal structure of the device by thermal drawing [80] of meters long tubing in consistent core geometries not available off-the-shelf today, followed by braiding of different angles interlocked around it.

Working fluid type. Furthermore, new material properties can be explored by using liquid media that allow for efficient hydraulic actuation [77]. Interactivity can be enhanced with responsive materials such as low melting point alloys, magnetic and supercritical fluids [34, 35, 45], and furthermore using biomaterials such as bodily fluids [2] as potential working fluids in a single OmniFiber device, or full body-worn system.

\section{CONCLUSION}

In this paper, we introduced OmniFiber, a reconfigurable material system based on fluidic fiber actuators. By functionalizing the fiber actuators with strain tunability, we achieve a wide range of movement-based I/O that we demonstrate through a variety of design cases. OmniFiber is built upon fluidic artificial muscle research, which originated from the field of soft robotics. These actuators are soft, and have high output performances (force/strain/speed). We expand on this basic actuator with technical improvements that together form a comprehensive fiber-based system with an integrated fabrication pipeline tailored towards the HCI audience. OmniFiber exhibits a range of morphing geometries including extension, contraction, bending, coiling, and micro-texture feedback

\footnotetext{
${ }^{5}$ Hagen-Poiseuille is a physical law that gives the pressure drop in an incompressible and Newtonian fluid in laminar flow flowing through a long cylindrical pipe of constant cross section.
} 
(Figure 2). Through our design space and applications, we demonstrate the rich potential of OmniFiber to benefit the research space of novel interactive materials on or close to the human body. As Qamar et. al pointed out in [55], "[..] the HCI community may benefit from a broader awareness of state-of-the-art material systems whereas the material science community has little experience in embedding information processing into structures... The tools needed for shaped-interface design need to be more expressive, like the raw and versatile materials an industrial designer might use to create complex geometries." OmniFiber contributes to closing this gap, adapting and shaping emerging materials to empower interaction designers. While there are many future challenges and design opportunities, we hope that the wide exploration of OmniFiber as an interaction design material and system opens a channel towards using the body as an intentional controller for movement-based HCI, leveraging sensing and actuating fiber-based systems.

\section{ACKNOWLEDGMENTS}

This work has been supported by Hardware for Energy Efficient Bodynets funded by the Swedish Foundation for Strategic Research project CHI19-0034. We thank the main author's spouse Atahan Afsar for his invaluable support at all stages of this work during the pandemic. The authors would also like to thank The Swedish School of Textiles for granting access to textile engineering labs; Erin Lewis and Nadia Campo Woytuk for advising on knitting techniques; Prof. Stefan Johansson for his professional advice on artificial muscles; Aishni Parab for the preliminary design tool; Priyanka Syal for the graphs; Kelsey Cotton for her connoisseurship on singing; Cedric Honnet for his time and feedback; Deema Qashat for proofreading; and Sanad Bushnaq for the rebuttal support.

\section{REFERENCES}

[1] Lea Albaugh, Scott Hudson, and Lining Yao. 2019. Digital fabrication of soft actuated objects by machine knitting. In Proceedings of the 2019 CHI Conference on Human Factors in Computing Systems. 1-13.

[2] Teresa Almeida, Madeline Balaam, Shaowen Bardzell, and Lone Koefoed Hansen. 2020. Introduction to the Special Issue on HCI and the Body: Reimagining Women's Health. ACM Trans. Comput.-Hum. Interact. 27, 4, Article 20 (Aug. 2020), 32 pages. https://doi.org/10.1145/3406091

[3] L. An, W.M. Huang, Y.Q. Fu, and N.Q. Guo. 2008. A note on size effect in actuating NiTi shape memory alloys by electrical current. Materials \& Design 29, 7 (2008), 1432-1437. https://doi.org/10.1016/j.matdes.2007.09.001

[4] Mahoro Anabuki and Hiroshi Ishii. 2007. Ar-jig: A handheld tangible user interface for modification of $3 \mathrm{~d}$ digital form via $2 \mathrm{~d}$ physical curve. In 2007 th IEEE and ACM International Symposium on Mixed and Augmented Reality. IEEE, 55-66.

[5] Erick Ball, Michael Meller, Jordan Chipka, and Ephrahim Garcia. 2016. Modeling and testing of a knitted-sleeve fluidic artificial muscle. Smart Materials and Structures 25 (11 2016), 115024. https://doi.org/10.1088/0964-1726/25/11/115024

[6] Priyanuj Bhuyan, Yuwen Wei, Dongho Sin, Jaesang Yu, Changwoon Nah, KwangUn Jeong, Michael Dickey, and Sungjune Park. 2021. Soft and Stretchable Liquid Metal Composites with Shape Memory and Healable Conductivity. ACS Applied Materials \& Interfaces 13 (06 2021). https://doi.org/10.1021/acsami.1c06786

[7] Moritz Bächer, Benjamin Hepp, Fabrizio Pece, Paul G. Kry, Bernd Bickel, Bernhard Thomaszewski, and Otmar Hilliges. 2016. DefSense: Computational Design of Customized Deformable Input Devices. In Proceedings of the 2016 CHI Conference on Human Factors in Computing Systems (CHI '16). Association for Computing Machinery, San Jose, California, USA, 3806-3816. https: //doi.org/10.1145/2858036.2858354

[8] Vito Cacucciolo, Hiroyuki Nabae, Koichi Suzumori, and Herbert Shea. 2020 Electrically-Driven Soft Fluidic Actuators Combining Stretchable Pumps With Thin McKibben Muscles. Frontiers in Robotics and AI 6 (2020). https://doi.org/ 10.3389/frobt.2019.00146 Publisher: Frontiers.

[9] Thomas P. Chenal, Jennifer C. Case, Jamie Paik, and Rebecca K. Kramer. 2014 Variable stiffness fabrics with embedded shape memory materials for wearable applications. In 2014 IEEE/RSf International Conference on Intelligent Robots and Systems. 2827-2831. https://doi.org/10.1109/IROS.2014.6942950

[10] Marcelo Coelho and Jamie Zigelbaum. 2011. Shape-changing interfaces. Personal and Ubiquitous Computing 15, 2 (2011), 161-173.

[11] Allen Cypher and Daniel Conrad Halbert. 1993. Watch what I do: programming by demonstration. MIT press.

[12] Alexandra Delazio, Ken Nakagaki, Roberta L Klatzky, Scott E Hudson, Jill Fain Lehman, and Alanson P Sample. 2018. Force jacket: Pneumatically-actuated jacket for embodied haptic experiences. In Proceedings of the 2018 CHI Conference on Human Factors in Computing Systems. 1-12.

[13] Roy Featherstone and Yee Harn Teh. 2006. Improving the Speed of Shape Memory Alloy Actuators by Faster Electrical Heating. In Experimental Robotics IX, Marcelo H. Ang and Oussama Khatib (Eds.). Springer Berlin Heidelberg, Berlin, Heidelberg, 67-76.

[14] Jack Forman, Taylor Tabb, Youngwook Do, Meng-Han Yeh, Adrian Galvin, and Lining Yao. 2019. ModiFiber: Two-Way Morphing Soft Thread Actuators for Tangible Interaction. 1-11. https://doi.org/10.1145/3290605.3300890

[15] Andreas Frutiger, Joseph Muth, Daniel Vogt, Yiğit Mengüç, Alexandre Campo, Alexander Valentine, Conor Walsh, and Jennifer Lewis. 2015. Capacitive Soft Strain Sensors via Multicore-Shell Fiber Printing. Advanced materials (Deerfield Beach, Fla.) 27 (03 2015). https://doi.org/10.1002/adma.201500072

[16] Oliver Glauser, Daniele Panozzo, Otmar Hilliges, and Olga Sorkine-Hornung. 2019. Deformation Capture via Soft and Stretchable Sensor Arrays. ACM Transactions on Graphics 38, 2 (March 2019), 16:1-16:16. https://doi.org/10.1145/3311972

[17] Carter Haines, Na Li, Geoffrey Spinks, Ali Aliev, Jiangtao Di, and Ray Baughman. 2016. New twist on artificial muscles. Proceedings of the National Academy of Sciences of the United States of America 113 (09 2016). https://doi.org/10.1073/ pnas. 1605273113

[18] Nur Al-huda Hamdan, Adrian Wagner, Simon Voelker, Jürgen Steimle, and Jan Borchers. 2019. Springlets: Expressive, Flexible and Silent On-Skin Tactile Interfaces. In Proceedings of the 2019 CHI Conference on Human Factors in Computing Systems (CHI '19). Association for Computing Machinery, Glasgow, Scotland Uk, 1-14. https://doi.org/10.1145/3290605.3300718

[19] Teng Han, Shubhi Bansal, Xiaochen Shi, Yanjun Chen, Baogang Quan, Feng Tian, Hongan Wang, and Sriram Subramanian. 2020. HapBead: On-Skin Microfluidic Haptic Interface using Tunable Bead. In Proceedings of the 2020 CHI Conference on Human Factors in Computing Systems. 1-10.

[20] John Hardy, Christian Weichel, Faisal Taher, John Vidler, and Jason Alexander. 2015. ShapeClip: Towards Rapid Prototyping with Shape-Changing Displays for Designers. In Proceedings of the 33rd Annual ACM Conference on Human Factors in Computing Systems (CHI '15). Association for Computing Machinery, Seoul, Republic of Korea, 19-28. https://doi.org/10.1145/2702123.2702599

[21] Kristina Höök, Caroline Hummels, Katherine Isbister, Patrizia Marti, Elena Márquez Segura, Martin Jonsson, Florian 'Floyd' Mueller, Pedro A.N. Sanches, Thecla Schiphorst, Anna Ståhl, Dag Svanaes, Ambra Trotto, Marianne Graves Petersen, and Youn-kyung Lim. 2017. Soma-Based Design Theory. In Proceedings of the 2017 CHI Conference Extended Abstracts on Human Factors in Computing Systems (Denver, Colorado, USA) (CHI EA '17). Association for Computing Machinery, New York, NY, USA, 550-557. https://doi.org/10.1145/3027063.3027082

[22] Caroline Hummels, Kees C. Overbeeke, and Sietske Klooster. 2007. Move to Get Moved: A Search for Methods, Tools and Knowledge to Design for Expressive and Rich Movement-Based Interaction. Personal Ubiquitous Comput. 11, 8 (Dec. 2007), 677-690. https://doi.org/10.1007/s00779-006-0135-y

[23] Pham Huy Nguyen and Wenlong Zhang. 2020. Design and Computational Modeling of Fabric Soft Pneumatic Actuators for Wearable Assistive Devices. Scientific Reports 10 (06 2020). https://doi.org/10.1038/s41598-020-65003-2

[24] Kristina Höök. 2018. Designing with the Body: Somaesthetic Interaction Design. https://doi.org/10.7551/mitpress/11481.001.0001

[25] Hiroshi Ishii, Dávid Lakatos, Leonardo Bonanni, and Jean-Baptiste Labrune. 2012. Radical atoms: beyond tangible bits, toward transformable materials. interactions 19, 1 (2012), 38-51.

[26] J. M. Jani, M. Leary, A. Subic, and M. Gibson. 2014. A review of shape memory alloy research, applications and opportunities. Materials \& Design 56 (2014), 1078-1113.

[27] Hsin-Liu Kao, Deborah Ajilo, Oksana Anilionyte, Artem Dementyev, Inrak Choi, Sean Follmer, and Chris Schmandt. 2017. Exploring interactions and perceptions of kinetic wearables. In Proceedings of the 2017 Conference on Designing Interactive Systems. 391-396.

[28] Tural Khudiyev, Jung Lee, Jason Cox, Eric Argentieri, Gabriel Loke, Rodger Yuan, Grace Noel, Ryoichi Tatara, Yang Yu, Frannie Logan, John Joannopoulos, Yang Shao-Horn, and Yoel Fink. 2020. $100 \mathrm{~m}$ Long Thermally Drawn Supercapacitor Fibers with Applications to 3D Printing and Textiles. Advanced Materials 32 (11 2020). https://doi.org/10.1002/adma.202004971

[29] Shoichiro Koizumi, Shunichi Kurumaya, Hiroyuki Nabae, Gen Endo, and Koichi Suzumori. 2020. Recurrent Braiding of Thin McKibben Muscles to Overcome Their Limitation of Contraction. Soft Robotics 7, 2 (2020), 251-258. https://doi. org/10.1089/soro.2019.0022 arXiv:https://doi.org/10.1089/soro.2019.0022 PMID: 31697196. 
[30] Shunichi Kurumaya, Hiroyuki Nabae, Gen Endo, and Koichi Suzumori. 2017. Design of thin McKibben muscle and multifilament structure. Sensors and Actuator A: Physical 261 (July 2017), 66-74. https://doi.org/10.1016/j.sna.2017.04.047

[31] Shunichi Kurumaya, Hiroyuki Nabae, Gen Endo, and Koichi Suzumori. 2019. Active Textile Braided in Three Strands with Thin McKibben Muscle. Soft Robotics 6, 2 (April 2019), 250-262. https://doi.org/10.1089/soro.2018.0076 Publisher: Mary Ann Liebert, Inc., publishers.

[32] Henry Lieberman. 2001. Your wish is my command: Programming by example. Morgan Kaufmann.

[33] W. Liu and C. R. Rahn. 2004. Fiber-Reinforced Membrane Models of McKibben Actuators . Journal of Applied Mechanics 70, 6 (01 2004), 853-859. https://doi.org/10.1115/1.1630812 arXiv:https://asmedigitalcollection.asme.org/appliedmechanics/articlepdf/70/6/853/5470497/853_1.pdf

[34] Qiuyu Lu, Chengpeng Mao, Liyuan Wang, and Haipeng Mi. 2016. Lime: Liquid metal interfaces for non-rigid interaction. In Proceedings of the 29th Annual Symposium on User Interface Software and Technology. 449-452.

[35] Qiuyu Lu, Jifei Ou, João Wilbert, André Haben, Haipeng Mi, and Hiroshi Ishii. 2019. milliMorph-Fluid-Driven Thin Film Shape-Change Materials for Interaction Design. In Proceedings of the 32nd Annual ACM Symposium on User Interface Software and Technology. 663-672.

[36] Ali Maziz, Alessandro Concas, Alexandre Khaldi, Jonas Stalhand, Nils-Krister Persson, and Edwin Jager. 2017. Knitting and weaving artificial muscles. Science Advances 3 (01 2017), e1600327. https://doi.org/10.1126/sciadv.1600327

[37] Seyed Mirvakili and Ian Hunter. 2017. Artificial Muscles: Mechanisms, Applications, and Challenges. Advanced Materials 30 (12 2017), 1704407. https: //doi.org/10.1002/adma.201704407

[38] Jaronie Mohd Jani, Martin Leary, Aleksandar Subic, and Mark A. Gibson. 2014. A review of shape memory alloy research, applications and opportunities. Materials \& Design (1980-2015) 56 (2014), 1078-1113. https://doi.org/10.1016/j.matdes.2013. 11.084

[39] Hila Mor, Tianyu Yu, Ken Nakagaki, Benjamin Harvey Miller, Yichen Jia, and Hiroshi Ishii. 2020. Venous Materials: Towards Interactive Fluidic Mechanisms. In Proceedings of the 2020 CHI Conference on Human Factors in Computing Systems. $1-14$.

[40] Sara Nabil, Jan Kučera, Nikoletta Karastathi, David S. Kirk, and Peter Wright. 2019 Seamless Seams: Crafting Techniques for Embedding Fabrics with Interactive Actuation. In Proceedings of the 2019 on Designing Interactive Systems Conference (San Diego, CA, USA) (DIS '19). Association for Computing Machinery, New York, NY, USA, 987-999. https://doi.org/10.1145/3322276.3322369

[41] Ken Nakagaki, Artem Dementyev, Sean Follmer, Joseph Paradiso, and Hiroshi Ishii. 2016. ChainFORM: A Linear Integrated Modular Hardware System for Shape Changing Interfaces. 87-96. https://doi.org/10.1145/2984511.2984587

[42] Ken Nakagaki, Sean Follmer, Artem Dementyev, Joseph A. Paradiso, and Hirosh Ishii. 2017. Designing Line-Based Shape-Changing Interfaces. IEEE Pervasive Computing 16, 4 (Oct. 2017), 36-46. https://doi.org/10.1109/MPRV.2017.3971127 Conference Name: IEEE Pervasive Computing.

[43] Ken Nakagaki, Sean Follmer, and Hiroshi Ishii. 2015. LineFORM: Actuated Curve Interfaces for Display, Interaction, and Constraint. In Proceedings of the 28th Annual ACM Symposium on User Interface Software \& Technology (UIST '15). Association for Computing Machinery, Charlotte, NC, USA, 333-339. https: //doi.org/10.1145/2807442.2807452

[44] Ken Nakagaki, Udayan Umapathi, Daniel Leithinger, and Hiroshi Ishii. 2017. AnimaStage: Hands-on Animated Craft on Pin-based Shape Displays. In Proceedings of the 2017 Conference on Designing Interactive Systems. 1093-1097.

[45] Kenichi Nakahara, Koya Narumi, Ryuma Niiyama, and Yoshihiro Kawahara 2017. Electric phase-change actuator with inkjet printed flexible circuit for printable and integrated robot prototyping. In 2017 IEEE International Conference on Robotics and Automation (ICRA). IEEE, 1856-1863.

[46] Ryosuke Nakayama, Ryo Suzuki, Satoshi Nakamaru, Ryuma Niiyama, Yoshihiro Kawahara, and Yasuaki Kakehi. 2019. MorphIO: Entirely Soft Sensing and Actuation Modules for Programming Shape Changes through Tangible Interaction. In Proceedings of the 2019 on Designing Interactive Systems Conference (DIS '19). Association for Computing Machinery, San Diego, CA, USA, 975-986. https://doi.org/10.1145/3322276.3322337

[47] Ryuma Niiyama, Xu Sun, Lining Yao, Hiroshi Ishii, Daniela Rus, and Sangbae Kim. 2015. Sticky Actuator: Free-Form Planar Actuators for Animated Objects. In Proceedings of the Ninth International Conference on Tangible, Embedded, and Embodied Interaction (Stanford, California, USA) (TEI '15). Association for Computing Machinery, New York, NY, USA, 77-84. https://doi.org/10.1145/2677199.2680600

[48] Takuya Nojima, Yoshiharu Ooide, and Hiroki Kawaguchi. 2013. Hairlytop interface: An interactive surface display comprised of hair-like soft actuators. In 2013 World Haptics Conference (WHC). 431-435. https://doi.org/10.1109/WHC.2013. 6548447

[49] Masaru Ohkubo and Takuya Nojima. 2018. SmartFiber: Reconfigurable Shape Changing Interface. In Proceedings of the 9th Augmented Human International Conference (AH '18). Association for Computing Machinery, Seoul, Republic of
Korea, 1-3. https://doi.org/10.1145/3174910.3174949

[50] Jifei Ou, Mélina Skouras, Nikolaos Vlavianos, Felix Heibeck, Chin-Yi Cheng, Jannik Peters, and Hiroshi Ishii. 2016. aeroMorph-heat-sealing inflatable shapechange materials for interaction design. In Proceedings of the 29th Annual Symposium on User Interface Software and Technology. 121-132.

[51] Joohee Park, Young-Woo Park, and Tek-Jin Nam. 2014. Wrigglo: shape-changing peripheral for interpersonal mobile communication. In Proceedings of the SIGCHI Conference on Human Factors in Computing Systems. 3973-3976.

[52] Sungjune Park, Neil Baugh, Hardil Shah, Dishit Parekh, Ishan Joshipura, and Michael Dickey. 2019. Ultrastretchable Elastic Shape Memory Fibers with Electrical Conductivity. Advanced Science 6 (11 2019), 1901579. https://doi.org/10. 1002/advs.201901579

[53] Amanda Parkes and Hiroshi Ishii. 2010. Bosu: a physical programmable design tool for transformability with soft mechanics. In Proceedings of the 8th ACM Conference on Designing Interactive Systems (DIS '10). Association for Computing Machinery, Aarhus, Denmark, 189-198. https://doi.org/10.1145/1858171.1858205

[54] Dietrich Parlitz, Thomas Peschel, and Eckart Altenmüller. 1998. Assessment of dynamic finger forces in pianists: Effects of training and expertise. Fournal of Biomechanics 31, 11 (1998), 1063-1067. https://doi.org/10.1016/S0021-9290(98) 00113-4

[55] Isabel P. S. Qamar, Rainer Groh, David Holman, and Anne Roudaut. 2018. HCI Meets Material Science: A Literature Review of Morphing Materials for the Design of Shape-Changing Interfaces. In Proceedings of the 2018 CHI Conference on Human Factors in Computing Systems (Montreal QC, Canada) (CHI '18). Association for Computing Machinery, New York, NY, USA, 1-23. https://doi.org/10.1145/ 3173574.3173948

[56] Eberhard Rabe, Hugo Partsch, Michael Jünger, Martin Abel, I Achhammer, François Becker, André Cornu-Thénard, Mie Flour, J Hutchinson, K Issberner, Ch Moffatt, and F Pannier. 2008. Guidelines for Clinical Studies with Compression Devices in Patients with Venous Disorders of the Lower Limb. European journal of vascular and endovascular surgery: the official journal of the European Society for Vascular Surgery 35 (05 2008), 494-500. https://doi.org/10.1016/j.ejvs.2007.08.006

[57] Hayes Solos Raffle, Amanda J. Parkes, and Hiroshi Ishii. 2004. Topobo: a constructive assembly system with kinetic memory. In Proceedings of the SIGCHI Conference on Human Factors in Computing Systems (CHI '04). Association for Computing Machinery, Vienna, Austria, 647-654. https://doi.org/10.1145/985692.985774

[58] Mohammad Rafiee, R. D. Farahani, and D. Therriault. 2020. MultiMaterial 3D and 4D Printing: A Survey. Advanced Science 7 (2020).

[59] Majken K Rasmussen, Esben W Pedersen, Marianne G Petersen, and Kasper Hornbæk. 2012. Shape-changing interfaces: a review of the design space and open research questions. In Proceedings of the SIGCHI Conference on Human Factors in Computing Systems. 735-744.

[60] Devin J. Roach, Chao Yuan, Xiao Kuang, Vincent Chi-Fung Li, Peter Blake, Marta Lechuga Romero, Irene Hammel, Kai Yu, and H. Jerry Qi. 2019. Long Liquid Crystal Elastomer Fibers with Large Reversible Actuation Strains for Smart Textiles and Artificial Muscles. ACS Applied Materials \& Interfaces 11, 21 (2019), 19514-19521. https://doi.org/10.1021/acsami.9b04401 arXiv:https://doi.org/10.1021/acsami.9b04401

[61] Vanessa Sanchez, Conor J. Walsh, and Robert J. Wood. [n.d.]. Textile Technology for Soft Robotic and Autonomous Garments. Advanced Functional Materials n/a, n/a ([n. d.]), 2008278. https://doi.org/10.1002/adfm.202008278 arXiv:https://onlinelibrary.wiley.com/doi/pdf/10.1002/adfm.202008278

[62] Benjamin Shih, Dylan Shah, Jinxing Li, Thomas G Thuruthel, Yong-Lae Park, Fumiya Iida, Zhenan Bao, Rebecca Kramer-Bottiglio, and Michael T Tolley. 2020. Electronic skins and machine learning for intelligent soft robots. (2020).

[63] Ali Shtarbanov. 2021. FlowIO Development Platform - the Pneumatic "Raspberry Pi” for Soft Robotics. Association for Computing Machinery, New York, NY, USA. https://doi.org/10.1145/3411763.3451513

[64] S. Sridar, C. J. Majeika, P. Schaffer, M. Bowers, S. Ueda, A. J. Barth, J. L. Sorrells, J. T. Wu, T. R. Hunt, and M. Popovic. 2016. Hydro Muscle -a novel soft fluidic actuator. In 2016 IEEE International Conference on Robotics and Automation (ICRA). 4014-4021.

[65] Yuta Sugiura, Calista Lee, Masayasu Ogata, Anusha Withana, Yasutoshi Makino, Daisuke Sakamoto, Masahiko Inami, and Takeo Igarashi. 2012. PINOKY: a ring that animates your plush toys. In Proceedings of the SIGCHI Conference on Human Factors in Computing Systems (CHI '12). Association for Computing Machinery, Austin, Texas, USA, 725-734. https://doi.org/10.1145/2207676.2207780

[66] Nobuhiro Takahashi, Shinichi Furuya, and Hideki Koike. 2020. Soft Exoskeleton Glove with Human Anatomical Architecture: Production of Dexterous Finger Movements and Skillful Piano Performance. IEEE Transactions on Haptics (2020).

[67] Nobuhiro Takahashi, Hayato Takahashi, and Hideki Koike. 2019. Soft exoskeleton glove enabling force feedback for human-like finger posture control with 20 degrees of freedom. In 2019 IEEE World Haptics Conference (WHC). IEEE, 217222

[68] Javier Tapia, Espen Knoop, Mojmir Mutný, Miguel A. Otaduy, and Moritz Bächer. 2019. MakeSense: Automated Sensor Design for Proprioceptive Soft Robots. Soft Robotics 7, 3 (Dec. 2019), 332-345. https://doi.org/10.1089/soro.2018.0162 Publisher: Mary Ann Liebert, Inc., publishers. 
[69] Jennifer R. A. Taylor and William M. Kier. 2003. Switching Skeletons: Hydrostatic Support in Molting Crabs. Science 301 5630 (2003), 209-210. https://doi.org/10.1126/science.1085987 arXiv:https://science.sciencemag.org/content/301/5630/209.full.pdf

[70] Shan-Yuan Teng, Tzu-Sheng Kuo, Chi Wang, Chi-huan Chiang, Da-Yuan Huang, Liwei Chan, and Bing-Yu Chen. 2018. Pupop: Pop-up prop on palm for virtual reality. In Proceedings of the 31st Annual ACM Symposium on User Interface Software and Technology. 5-17.

[71] Rashi Tiwari, Michael A Meller, Karl B Wajcs, Caris Moses, Ismael Reveles, and Ephrahim Garcia. 2012. Hydraulic artificial muscles. fournal of Intelligent Material Systems and Structures 23, 3 (2012), 301-312. https://doi.org/10.1177/ 1045389X12438627 arXiv:https://doi.org/10.1177/1045389X12438627

[72] Jonas Togler, Fabian Hemmert, and Reto Wettach. 2009. Living interfaces: the thrifty faucet. In Proceedings of the 3rd International Conference on Tangible and Embedded Interaction. 43-44.

[73] Marynel Vázquez, Eric Brockmeyer, Ruta Desai, Chris Harrison, and Scott E Hudson. 2015. 3d printing pneumatic device controls with variable activation force capabilities. In Proceedings of the 33rd Annual ACM Conference on Human Factors in Computing Systems. 1295-1304.

[74] Akira; Akito Nakano and Michihiko Ueno Wakita. 2011. SMAAD Surface: A tangible interface for smart material aided architectural design. In Proceedings of the 16th International Conference on Computer Aided Architectural Design Research in Asia / The University of Newcastle, Australia 27-29 April 2011, pp. 355-364. CUMINCAD. http://papers.cumincad.org/cgi-bin/works/BrowseTree= series=AZ/Show?caadria2011_034

[75] Guanyun Wang, Ye Tao, Ozguc Bertug Capunaman, Humphrey Yang, and Lining Yao. 2019. A-line: 4D Printing Morphing Linear Composite Structures. In Proceedings of the 2019 CHI Conference on Human Factors in Computing Systems. $1-12$.

[76] Mark Weiser. 1999. The computer for the 21st century. ACM SIGMOBILE mobile computing and communications review 3, 3 (1999), 3-11.

[77] Chaoqun Xiang, Maria Elena Giannaccini, Theo Theodoridis, Lina Hao, Samia Nefti-Meziani, and Steven Davis. 2016. Variable stiffness Mckibben muscles with hydraulic and pneumatic operating modes. Advanced Robotics 30, 13 (2016), 889-899. https://doi.org/10.1080/01691864.2016.1154801 arXiv:https://doi.org/10.1080/01691864.2016.1154801
[78] Lining Yao, Ryuma Niiyama, Jifei Ou, Sean Follmer, Clark Della Silva, and Hiroshi Ishii. 2013. PneUI: pneumatically actuated soft composite materials for shape changing interfaces. In Proceedings of the 26th annual ACM symposium on User interface software and technology (UIST '13). Association for Computing Machinery, St. Andrews, Scotland, United Kingdom, 13-22. https: //doi.org/10.1145/2501988.2502037

[79] Hong Kai Yap, Jeong Hoon Lim, Fatima Nasrallah, James CH Goh, and Raye CH Yeow. 2015. A soft exoskeleton for hand assistive and rehabilitation application using pneumatic actuators with variable stiffness. In 2015 IEEE international conference on robotics and automation (ICRA). IEEE, 4967-4972.

[80] Rodger Yuan, Jaemyon Lee, Hao-Wei Su, Etgar Levy, Tural Khudiyev, Joel Voldman, and Yoel Fink. 2018. Microfluidics in structured multimaterial fibers. Proceedings of the National Academy of Sciences 115 (10 2018), 201809459. https: //doi.org/10.1073/pnas.1809459115

[81] Taigo Yukisawa, Yasuaki Ishii, Satoshi Nishikawa, Ryuma Niiyama, and Yasuo Kuniyoshi. 2017. Modeling of extensible pneumatic actuator with bellows (EPAB) for continuum arm. In 2017 IEEE International Conference on Robotics and Biomimetics (ROBIO). 2303-2308. https://doi.org/10.1109/ROBIO.2017.8324762

[82] Jun Zhang, Jun Sheng, Ciarán T O’Neill, Conor J Walsh, Robert J Wood, Jee-Hwan Ryu, Jaydev P Desai, and Michael C Yip. 2019. Robotic artificial muscles: Curren progress and future perspectives. IEEE Transactions on Robotics 35, 3 (2019), 761-781.

[83] Zhihao Zhou, K. Chen, X. Li, Songlin Zhang, Yu fen Wu, Yihao Zhou, Keyu Meng, Chenchen Sun, Q. He, W. Fan, Endong Fan, Zhiwei Lin, Xulong Tan, W. Deng, J. Yang, and Junzhang Chen. 2020. Sign-to-speech translation using machine-learning-assisted stretchable sensor arrays. Nature Electronics 3 (2020), 571-578.

[84] Kening Zhu and Shengdong Zhao. 2013. AutoGami: a low-cost rapid prototyping toolkit for automated movable paper craft. In Proceedings of the SIGCHI Conference on Human Factors in Computing Systems (CHI '13). Association for Computing Machinery, Paris, France, 661-670. https://doi.org/10.1145/2470654.2470748

[85] Mengjia Zhu, Amirhossein H. Memar, Aakar Gupta, Majed Samad, Priyanshu Agarwal, Yon Visell, Sean J. Keller, and Nicholas Colonnese. 2020. PneuSleeve: In-fabric Multimodal Actuation and Sensing in a Soft, Compact, and Expressive Haptic Sleeve. In Proceedings of the 2020 CHI Conference on Human Factors in Computing Systems (CHI '20). Association for Computing Machinery, Honolulu, HI, USA, 1-12. https://doi.org/10.1145/3313831.3376333 